\title{
INVERSE SPECTRAL RESULTS ON EVEN DIMENSIONAL TORI.
}

\author{
CAROLYN S. GORDON, PIERRE GUERINI, THOMAS KAPPELER, AND DAVID L. WEBB
}

\section{CONTENTS}

1. Introduction.

2. Line bundles over tori: construction and classification. 3

3. Inverse spectral results on rectangular tori. 12

4. Tori with nondegenerate length spectrum. 16

Appendix A. Wave kernel on $\mathbb{R}^{n}$. 19

Appendix B. Wave kernel acting on sections of a line bundle.

Appendix C. Expansion of the wave trace at singularities.

Appendix D. Proof of Proposition C.11. 30

References

\section{INTRODUCTION.}

Let $M$ be a flat $n$-dimensional torus $\mathcal{L} \backslash \mathbb{R}^{n}$. To each smooth function $Q$ on $M$, viewed as an $\mathcal{L}$-periodic function $Q: \mathbb{R}^{n} \rightarrow \mathbb{R}$, one associates a Schrödinger operator $\Delta+Q$. In their pioneering work [E-R-T], G. Eskin, J. Ralston, and E. Trubowitz address the extent to which the potential $Q$ is determined by its periodic spectrum $\operatorname{Spec}(Q)$, i.e., by the spectrum of the Schrödinger operator acting on $\mathcal{L}$-periodic functions, and also by the more extensive data of the Bloch spectrum. The latter associates to each linear functional $\alpha$ on $\mathbb{R}^{n}$ the spectrum $\operatorname{Spec}_{\alpha}(Q)$ of the Schrödinger operator acting on smooth functions on $\mathbb{R}^{n}$ satisfying the condition $f(x+l)=e^{2 \pi i \alpha(l)} f(x)$ for all $l \in \mathcal{L}$. In what follows, we will refer to the Bloch spectrum as the "classical Bloch spectrum". As explained in Section 2, the classical Bloch spectrum can be viewed as the spectrum of the bundle Laplacian determined by a connection on the trivial complex line bundle over $M$.

Given a Hermitian line bundle $L$ over $M$ and a connection $\nabla$ on $L$, one associates a Laplace operator acting on smooth sections of $L$. A smooth function $Q$ on $M$ then gives rise to a Schrödinger operator acting on sections of the bundle. The spectrum $\operatorname{Spec}(Q ; L, \nabla)$ of this operator depends both on the connection and on the potential. In case $L$ is the trivial bundle and $\nabla$ the trivial connection, then $\operatorname{Spec}(Q ; L, \nabla)$ coincides with the periodic spectrum of $Q$. The torus $M=\mathcal{L} \backslash \mathbb{R}^{n}$ is said to have nondegenerate length spectrum if for each $l \in \mathcal{L}$, the only elements of $\mathcal{L}$ of length $|l|$ are $\pm l$. For line bundles of Chern number one over two-dimensional tori, V. Guillemin [G] addressed the question of whether the spectrum determines both the potential and the connection. He obtained an affirmative answer in the case of 2-tori with nondegenerate length spectrum, provided that both the potential and the connection are invariant under the isometry of $M$ defined by the map $x \rightarrow-x$ of $\mathbb{R}^{2}$ and that the connection satisfies suitable curvature bounds.

In this article, we consider Schrödinger operators on Hermitian line bundles $L$ over higher-dimensional tori. In general dimensions, it seems unlikely that one could recover the connection from the spectrum of the Schrödinger operator. Thus we fix either a single connection or a family of specified connections on a line bundle $L$ and ask whether the potential function can be determined from the associated spectral data. Associated to each connection

Guerini and Kappeler were supported in part by the Swiss National Science Foundation and by the European Research Training Network HPRN-CT-1999-00118; Gordon and Webb were supported in part by NSF grants DMS-0306752. 
on $L$ is a curvature 2-form on $M$. By an abuse of language, we will refer to a connection as translation-invariant if the associated curvature 2-form is translation-invariant, i.e., invariant under the group of isometries of the torus given by the translations. For a given potential function $Q$, the mapping $\nabla \mapsto \operatorname{Spec}(Q ; L, \nabla)$ as $\nabla$ ranges over the translation-invariant connections is a natural analog of the classical Bloch spectrum (as explained in Remark 2.38) and thus will be referred to as the $L$-Bloch spectrum of $Q$, or simply as the Bloch spectrum of $Q$ if $L$ is understood. We will also distinguish a particular translation-invariant connection $\nabla^{D}$ on $L$ and will use the simplified notation $\operatorname{Spec}(Q ; L)$ for $\operatorname{Spec}\left(Q ; L, \nabla^{D}\right)$. The set of all potentials $P$ for which $\operatorname{Spec}(P ; L)=$ $\operatorname{Spec}(Q ; L)$ will be denoted $I \operatorname{so}(Q ; L)$. As will be explained in Section 2, our choice of a distinguished leftinvariant connection $\nabla^{D}$ allows us to parameterize the translation-invariant connections by the harmonic 1-forms on $M$ or, equivalently, by linear functionals $\alpha$ on $\mathbb{R}^{n}$. The distinguished connection corresponds to $\alpha=0$. We set $\operatorname{Spec}_{\alpha}(Q ; L)=\operatorname{Spec}\left(Q ; L, \nabla_{\alpha}\right)$. Note that the harmonic 1-forms on $M$ may be viewed as linear functionals on $\mathbb{R}^{n}$.

A line bundle over a torus is uniquely determined up to equivalence by its Chern class. We consider bundles whose Chern class is represented by a nondegenerate two-form. This condition forces the dimension of $M$ to be even, say $n=2 m$. As will be explained in Section 2, associated to the Chern class is an ordered $m$-tuple $\left(r_{1}, \ldots, r_{m}\right)$ of positive integers such that $r_{i} \mid r_{i+1}$ for $i=1, \ldots, m-1$; we refer to the integers $r_{j}$ as the invariant factors of the Chern class. The $m$-tuple of invariant factors is a topological invariant of the line bundle but not a complete invariant (although in the case $m=1, r_{1}$ is, up to sign, the Chern number of the bundle).

We obtain positive results in the generic case of tori with nondegenerate length spectrum and negative results (counterexamples) in the case of rectangular tori. The counterexamples show that, both in the two-dimensional case studied by Guillemin and in the higher dimensional case studied here, the hypothesis of nondegeneracy of the length spectrum cannot be dropped.

We first state the negative results. Two potentials $Q_{1}$ and $Q_{2}$ on a torus $M$ are said to be congruent if there exists an isometry $\sigma$ of $M$ such that $Q_{2}=Q_{1} \circ \sigma$.

1.1. THEOREM. Let $M$ be a rectangular nonsquare 2-torus for which the ratio of the side lengths is rational. Then for every line bundle $L$ over $M$ with nondegenerate Chern class, there exists a pair of noncongruent potentials on $M$ that are isospectral with respect to the Schrödinger operator defined by the distinguished invariant connection on $L$.

A slightly weaker result holds in arbitrary even dimensions, as explained in Section 3.

1.2. REMARK. We actually prove a somewhat stronger statement than that given in Theorem 1.1. For simplicity, we state the stronger version in a special case: Let $\mathcal{L}$ be the lattice $2 \mathbb{Z} \times \frac{1}{2} \mathbb{Z}$ in $\mathbb{R}^{2}$, let $M=\mathcal{L} \backslash \mathbb{R}^{2}$, and let $L \rightarrow M$ be a line bundle with Chern number $r_{1}=1$. Given any smooth function $h: \mathbb{R} \rightarrow \mathbb{R}$ of period $\frac{1}{2}$, define potentials $Q_{1}$ and $Q_{2}$ on $M$ by $Q_{1}(x, y)=h(x)$ and $Q_{2}(x, y)=h(y)$. Then $\operatorname{Spec}\left(Q_{1} ; L\right)=\operatorname{Spec}\left(Q_{2} ; L\right)$.

In contrast, according to Theorem 4.2 in [Go-K1], the potentials in Remark 1.2 do not have the same periodic spectrum. This example is contrary to the general belief that "spectral rigidity" in the case of a non trivial line bundle is more pronounced than in the case of a trivial line bundle.

The method used to construct the examples in Theorem 1.1 also yield results concerning the spectrum of the Laplace operator itself. By the spectrum of a line bundle $L$ over a flat torus $M$, we will mean the spectrum of the Laplace operator associated with the distinguished invariant connection on $L$. We show:

- For every even integer $n \geq 4$, there exists an $n$-dimensional flat rectangular torus $M$ and a pair of topologically distinct, isospectral line bundles over $M$. The Chern classes of these line bundles have different invariant factors.

- For every even integer $n=2 m$, there exist pairs of nonisometric $n$-dimensional tori $M$ and $M^{\prime}$ such that for every choice of $r=\left(r_{1}, \ldots, r_{m}\right)$ as above, there is a pair of isospectral line bundles $L_{r} \rightarrow M$ and $L_{r}^{\prime} \rightarrow M^{\prime}$. Thus the spectrum does not determine the isometry class of the torus.

We next address the positive results. First note that the spectrum of the Schrödinger operator associated with a fixed connection on a line bundle $L$ may sometimes distinguish between congruent potentials. In particular, the 
map $x \rightarrow-x$ of $\mathbb{R}^{n}$ gives rise to an isometric action of $\mathbb{Z}_{2}$ on $M$. Given a smooth potential $Q$ on $M$, let $\check{Q}$ denote the congruent potential given by $\check{Q}(x)=Q(-x)$. For the bundles $L$ that we consider, $\operatorname{Spec}(\check{Q} ; L, \nabla)$ will typically differ from $\operatorname{Spec}(Q ; L, \nabla)$ when $\nabla$ is not $\mathbb{Z}_{2}$-invariant. In fact, a consequence of Theorem 1.4 below is that $Q$ and $\check{Q}$ are distinguished by their $L$-Bloch spectra when all the Chern invariant factors of $L$ equal one. On the other hand, $\operatorname{Spec}(\check{Q} ; L, \nabla)$ and $\operatorname{Spec}(Q ; L, \nabla)$ do agree when $\nabla$ is $\mathbb{Z}_{2}$-invariant, in particular, when $\nabla=\nabla^{D}$.

Our first theorem below generalizes to tori $M$ of arbitrary even dimension $n=2 m$ a result of Guillemin [G] in dimension two. Both theorems below are based on wave invariants constructed in the appendix.

1.3. TheOREM. Let $M^{2 m}$ be an even-dimensional torus with nondegenerate length spectrum, and let $L \rightarrow M$ be a line bundle with Chern invariant factors $r_{1}=\cdots r_{m}=1$. Then the following statements hold:

(a) For any $P \in I s o(Q ; L)$,

$$
\frac{1}{2}(P+\check{P})=\frac{1}{2}(Q+\check{Q})
$$

i.e. the even part $Q^{+}:=\frac{1}{2}(Q+\check{Q})$ of $Q$ is uniquely determined by $\operatorname{Spec}(Q ; L)$.

(b) If $Q$ is assumed to be even, then Iso $(Q ; L)=\{Q\}$.

Both statements remain true when the distinguished connection on $L$ is replaced by any connection which is both translation-invariant and $\mathbb{Z}_{2}$-invariant.

1.4. TheOREM. Assume that $M^{2 m}$ has nondegenerate length spectrum and $L \rightarrow M$ is a line bundle with Chern invariant factors $r_{1}=\cdots r_{m}=1$. Then every potential $Q$ is uniquely determined by its $L$-Bloch spectrum. In fact, if $\alpha$ and $\beta$ are any two linear functionals on $\mathbb{R}^{n}$ satisfying the generic property that $(\alpha-\beta)(l) \notin \mathbb{Q}$ for any nonzero $l \in \mathcal{L}$, then the spectra $\operatorname{Spec}_{\alpha}(Q ; L)$ and $\operatorname{Spec}_{\beta}(Q ; L)$ together suffice to determine $Q$.

We will show in Section 4 that when $m=1$, the odd part of $Q$ has strong spectral rigidity properties as well (see Theorem 4.12).

Since, as noted above, $\operatorname{Spec}(Q ; L)=\operatorname{Spec}(\check{Q} ; L)$, Theorem 1.4 shows that $\operatorname{Spec}(Q ; L)$ does not suffice to determine the full $L$-Bloch spectrum of $Q$. Theorem 1.4 fits nicely with results of the third author $[\mathrm{K}]$ for a discrete version of the Schrödinger equation on a 2-dimensional torus. There it was shown that, generically, the periodic spectrum does not determine the Bloch spectrum of $Q$ but, again generically, two $\operatorname{spectra} S p e c_{\alpha}(Q)$ and $\operatorname{Spec}_{\beta}(Q)$ do.

In contrast, when the bundle $L$ in Theorem 1.4 is replaced by a trivial bundle, then the conclusion fails. In fact, it can be easily verified that $Q$ and $\check{Q}$ have the same classical Bloch spectrum. (See Remark 4.6.) Moreover, G. Eskin, J. Ralston, and E. Trubowitz [E-R-T] showed that for real analytic potentials $Q$, the periodic spectrum of $Q$ determines the classical Bloch spectrum of $Q$. While we have seen that their conclusion fails in our setting, we do nonetheless have that $\operatorname{Spec}_{\alpha}(\check{Q}, L)=\operatorname{Spec}_{-\alpha}(Q, L)$. (Again, see Remark 4.6.) Hence one may ask whether, say for real analytic potentials, $\operatorname{Spec}(Q ; L)$ determines each pair $\left(\operatorname{Spec}_{\alpha}(Q ; L), \operatorname{Spec}_{-\alpha}(Q ; L)\right)$ up to order.

The paper is organized as follows. In section 2 we introduce the line bundles, connections and spectra as well as the notation that are used throughout of the paper. The reader interested only in wave invariants and positive results need only read the first two subsections of Section 2. The third subsection gives the background used in the construction of the counterexamples. In section 3 we prove Theorem 1.1 and other negative inverse spectral results for rectangular tori. In section 4 we use wave invariants to prove Theorem 1.3, Theorem 1.4 and other positive results. In the appendices, we construct the necessary wave invariants by adapting to our setting constructions developed in [E-R-T] as well as in [E] and [G].

\section{LINE BUNDLES OVER TORI: CONSTRUCTION AND CLASSIFICATION.}

\section{A. Invariant factors of 2-forms.}

Let $n \geq 2$, let $\mathcal{L}$ be a lattice of maximal rank in $\mathbb{R}^{n}$, and let $g$ be an inner product on $\mathbb{R}^{n}$. Then $M:=\left(\mathcal{L} \backslash \mathbb{R}^{n}, g\right)$ is a flat Riemannian torus of dimension $n$. We review the classification and construction of complex line bundles over $M$.

A complex line bundle $L$ over $M$ is classified up to equivalence by its Chern class $c_{1}(L)$ in $H^{2}(M ; \mathbb{Z})$. Like every real or integer cohomology class on the torus $M$, the Chern class may be uniquely represented by a translation 
invariant 2-form $\Omega$; this is the harmonic representative of the class. This form $\Omega$ may be viewed as an antisymmetric bilinear map on $\mathbb{R}^{n} \times \mathbb{R}^{n}$ that takes integer values on $\mathcal{L} \times \mathcal{L}$. We will consider only bundles with nondegenerate Chern class; i.e., we require that $\Omega$ be a nondegenerate bilinear map. In order that such a nondegenerate antisymmetric bilinear form exist, the dimension $n$ of $M$ must be even.

The following result is standard. See, for example, [GH], page 304.

2.1. LEMMA. Let $n=2 m$ be an even integer. Given a nondegenerate antisymmetric bilinear map $\Omega$ on $\mathbb{R}^{n} \times \mathbb{R}^{n}$ that takes integer values on $\mathcal{L} \times \mathcal{L}$, there exists a unique $m$-tuple $\left(r_{1}, \ldots, r_{m}\right)$ of positive integers with the following properties:

- $r_{1}\left|r_{2}\right| \ldots \mid r_{m}$;

- There exists a lattice basis $\mathcal{B}=\left\{U_{1}, \ldots, U_{m}, V_{1}, \ldots, V_{m}\right\}$ of $\mathcal{L}$ such that $\Omega\left(U_{j}, V_{j}\right)=r_{j}$ for $j=$ $1, \ldots, m$ and $\Omega$ vanishes on all other pairs of vectors from $\mathcal{B}$.

Equivalently, letting $(u, v):=\left(u_{1}, \ldots, u_{m}, v_{1}, \ldots, v_{m}\right)$ be the coordinates on $\mathbb{R}^{n}$ associated with the basis $\mathcal{B}$ and viewing $\Omega$ as a 2 -form on $\mathbb{R}^{n}$, we have

$$
\Omega=\sum_{j=1}^{m} r_{j} d u_{j} \wedge d v_{j} .
$$

2.2. REMARK. The nondegenerate form $\Omega: \mathcal{L} \times \mathcal{L} \rightarrow \mathbb{Z}$ induces an injective map $\mathcal{L} \rightarrow \mathcal{L}^{*}:=\operatorname{Hom}_{\mathbb{Z}}(\mathcal{L}, \mathbb{Z})$, i.e., an injection $\mathbb{Z}^{n} \rightarrow \mathbb{Z}^{n}$. The sequence $r_{1}, r_{1}, r_{2}, r_{2}, \ldots, r_{m}, r_{m}$ is just the sequence of invariant factors associated with this injection, i.e., the entries of its Smith normal form.

2.3. Definition. In view of Remark 2.2, in the notation of Lemma 2.1, we will refer to the integers $\left(r_{1}, \ldots, r_{m}\right)$ as the invariant factors of the cohomology class $[\Omega] \in H^{2}(M ; \mathbb{Z})$.

2.4. NotATiOn.

(i) Throughout the rest of this section, we fix $r=\left(r_{1}, \ldots, r_{m}\right)$ (all nonzero) with $r_{1}\left|r_{2}\right| \ldots \mid r_{m}$, and we fix a basis $\mathcal{B}=\left\{U_{1}, \ldots, U_{m}, V_{1}, \ldots, V_{m}\right\}$, thus defining coordinates $\left(u_{1}, \ldots, u_{m}, v_{1}, \ldots, v_{m}\right)$. For $x \in \mathbb{R}^{n}$, we will express $x$ in these coordinates as $x=(u(x), v(x))=\sum_{j=1}^{m} u_{j}(x) U_{j}+v_{j}(x) V_{j}$. Define a 2-form $\Omega$ by $\Omega=\sum_{j=1}^{m} r_{j} d u_{j} \wedge d v_{j}$.

(ii) Given $x, y$ in $\mathbb{R}^{n}$, define

$$
e_{x}(y)=\sum_{j=1}^{m} r_{j} u_{j}(x) v_{j}(y) .
$$

In particular, viewing $\Omega$ as a bilinear form on $\mathbb{R}^{n}$, we have $\Omega(x, y)=e_{x}(y)-e_{y}(x)$.

2.6. REMARK. In the notation of $2.4,[\Omega]$ depends on the choice of $\mathcal{B}$ as well as on $\left(r_{1}, \ldots, r_{m}\right)$. However, if the torus $M$ is two-dimensional, the cohomology class depends only on the invariant factor $r\left(=r_{1}\right)$ and the orientation of the basis $\mathcal{B}$. In this case, if $L$ is a line bundle with Chern class $c_{1}(L)=[\Omega]$, then the Chern number of $L$ is $\pm r$, with the sign depending on the orientation of the basis $\mathcal{B}$. While we will normally assume that the integers $r_{i}$ in 2.4 are positive (i.e., that they are the invariant factors as defined in 2.3), the constructions in Subsections 2B and $2 \mathrm{C}$ below never use the positivity of the integers $r_{i}$. In considering two-dimensional tori, it is convenient to allow $r$ to be an arbitrary nonzero integer so that as $r$ varies (with the basis $\mathcal{B}$ remaining fixed), the corresponding 2-forms $\Omega$ range over all nontrivial cohomology classes in $H^{2}(M ; \mathbb{Z})$, which parametrize nontrivial complex line bundles over a two-dimensional torus $M$.

\section{B. Line bundles, connections, and spectra.}

Given the data in Notation 2.4(i), we now construct a complex Hermitian line bundle $L$ over the torus $M$ and a connection on $L$. By computing the curvature of this connection, we will see below that the line bundle has Chern class $[\Omega]$. 
2.7. Definition. Define an action of the group $\mathcal{L}$ on the total space $\mathbb{R}^{n} \times \mathbb{C}$ of the trivial complex line bundle over $\mathbb{R}^{n}$ as follows: for $l \in \mathcal{L}, x \in \mathbb{R}^{n}$, and $z \in \mathbb{C}$,

$$
l .(x, z)=\left(l+x, e^{2 \pi i e_{l}(x)} z\right),
$$

where $e_{l}(x)$ is given by Notation 2.4(ii). We define $L$ to be the orbit space of the $\mathcal{L}$-action on $\mathbb{R}^{n} \times \mathbb{C}$.

Denote elements of $L$ by $[(x, z)]$. The bundle projection $\mathbb{R}^{n} \times \mathbb{C} \rightarrow \mathbb{R}^{n}$ is $\mathcal{L}$-equivariant, so induces $\pi: L \rightarrow M$ by $\pi([(x, z)])=\bar{x} \in \mathcal{L} \backslash \mathbb{R}^{n}=M$. Then $L$ is a complex line bundle over $M$. Moreover, $L$ to our original trivial bundle $\mathbb{R}^{n} \times \mathbb{C}$ over $\mathbb{R}^{n}$. Many of our considerations - sections, connections, curvature, etc. - will be simplified by expressing data associated with the bundle $L$ via corresponding "pullback" data associated with the trivial complex line bundle over $\mathbb{R}^{n}$.

(Aside: We can endow $L$ with local trivializations in such a way that its structure group is the unitary group $U(1)$. To describe the local trivializations and transition functions explicitly, let $p: \mathbb{R}^{n} \rightarrow M=\mathcal{L} \backslash \mathbb{R}^{n}$ be the projection. Given $\bar{x} \in M$, let $\bar{U}$ be an evenly covered neighborhood of $\bar{x}$ in $M$, so $p^{-1}(\bar{U})$ is a union of disjoint open sheets each carried diffeomorphically by $p$ to $\bar{U}$. Let $U$ be one of these sheets. For each $\bar{y} \in \bar{U}$, let $y \in U$ be the unique lift of $\bar{y}$ in $U$. Define $t_{U}:\left.L\right|_{U} \rightarrow \bar{U} \times \mathbb{C}$ by $t_{U}([(y, z)])=(\bar{y}, z)$. If $U^{\prime}$ is another of the sheets of $p^{-1}(\bar{U})$, then there exists $l \in \mathcal{L}$ such that $U^{\prime}=l+U=\{l+y: y \in U\}$. The transition function $t_{U^{\prime}} \circ\left(t_{U}\right)^{-1}: \bar{U} \times \mathbb{C} \rightarrow \bar{U} \times \mathbb{C}$ is given by $(\bar{y}, z) \mapsto\left(\bar{y}, \sigma_{U^{\prime} U}(\bar{y}) z\right)$, where $\sigma_{U^{\prime} U}: \bar{U} \cap \bar{U}^{\prime} \rightarrow U(1)$ is given by $\bar{y} \mapsto e^{2 \pi i e_{l}(y)}$. We emphasize that $e^{2 \pi i e_{l}(y)}$ is independent of the choice of lift $y$ of $\bar{y}$ in $\mathbb{R}^{n}$ since $e_{l}(\mathcal{L}) \subset \mathbb{Z}$. $)$

The Hermitian structure on the trivial bundle $\mathbb{R}^{n} \times \mathbb{C}$ given by the standard Hermitian inner product on $\mathbb{C}$ induces a Hermitian structure on $L$.

Given any bundle $B$, we denote by $\mathcal{E}(B)$ the space of smooth sections of $B$. An element of $\mathcal{E}\left(\mathbb{R}^{n} \times \mathbb{C}\right)$ is a map $s: \mathbb{R}^{n} \rightarrow \mathbb{R}^{n} \times \mathbb{C}$ of the form $s(x)=(x, h(x))$ for some smooth complex valued function $h$ on $\mathbb{R}^{n}$. The group $\mathcal{L}$ acts on $\mathcal{E}\left(\mathbb{R}^{n} \times \mathbb{C}\right)$ on the left via the action

$$
(l\lrcorner s)(x)=l \cdot(s(x-l))=\left(x, e^{2 \pi i e_{l}(x)} h(x-l)\right) .
$$

Sections of $L$ pull back to sections $s: \mathbb{R}^{n} \rightarrow \mathbb{R}^{n} \times \mathbb{C}$ of the trivial bundle that are $\mathcal{L}$-invariant for this action, i.e., to sections that satisfy

$$
s(l+x)=l . s(x)
$$

for all $l \in \mathcal{L}$ and $x \in \mathbb{R}^{n}$. Such a section has the form

$$
s(x)=(x, h(x))
$$

with $h$ a complex-valued function on $\mathbb{R}^{n}$ satisfying the functional equation

$$
h(l+x)=e^{2 \pi i e_{l}(x)} h(x)
$$

for $x \in \mathbb{R}^{n}$ and $l \in \mathcal{L}$. Given such a function $h$, the corresponding section $\bar{h}$ of $L$ is given by

$$
\bar{h}(\bar{x})=[(x, h(x))] .
$$

2.14. Definition. Given a complex-valued 1 -form $A$ on $\mathbb{R}^{n}$, we obtain a connection $\nabla$ on the trivial bundle $\mathbb{R}^{n} \times \mathbb{C}$ over $\mathbb{R}^{n}$ by

$$
\nabla=d+A
$$

Thus for a section $s$ given by $s(x)=(x, h(x))$ and a tangent vector $X \in T_{x} \mathbb{R}^{n}$,

$$
\left(\nabla_{X} s\right)(x)=\left(x, X(h)+A_{x}(X) h(x)\right) .
$$

The connection $\nabla$ descends to a connection on $L$ precisely if it carries $\mathcal{L}$-invariant sections to $\mathcal{L}$-invariant sections. More precisely, the condition that $\nabla$ descend to a connection on $L$ is the following: if $s$ is a section of the trivial complex line bundle over $\mathbb{R}^{n}$ satisfying (2.10), then for every $X \in T_{x} \mathbb{R}^{n}$ and $l \in \mathcal{L}$, we have that

$$
\left(\nabla_{T_{l *}(X)} s\right)(l+x)=l \cdot\left(\nabla_{X} s\right)(x)
$$


where $T_{l}: \mathbb{R}^{n} \rightarrow \mathbb{R}^{n}$ denotes translation by $l$. In this case we will use the same notation $\nabla$ for the connection thus obatined on $L$.

One checks that the condition on $A$ that guarantees that the connection descend in this way to a connection on the line bundle $L$ over the torus is that, for any $l \in \mathcal{L}$,

$$
T_{l}^{*} A=A-2 \pi i e_{l}
$$

(one uses the fact that $e_{l}$ can be viewed as either a function or a 1-form, and for a tangent vector $X \in T_{x} \mathbb{R}^{n}$, $\left.X\left(e_{l}\right)=\left.e_{l}\right|_{x}(X)\right)$.

A necesssary and sufficient condition that the connection be compatible with the Hermitian structure on the bundle is that the 1-form $A$ be purely imaginary; for convenience, we will write $A=2 \pi i \varphi$ for some real-valued 1 -form $\varphi$ on $\mathbb{R}^{n}$. We will assume in what follows that all connections are Hermitian.

2.17. REMARK. For $l \in \mathcal{L}$, view the linear functional $e_{l}$ as a harmonic (equivalently, translation-invariant) 1form on $\mathbb{R}^{n}$. Then by Equation (2.16), a connection $\nabla=d+2 \pi i \varphi$ satisfies Equation (2.15), and thus defines a connection on $L$, if and only if $T_{l}^{*} \varphi=\varphi-e_{l}$ for all $l \in \mathcal{L}$. By identifying each tangent space $T_{x} \mathbb{R}^{n}$ with $\mathbb{R}^{n}$ and thus viewing the covectors $\left.\varphi\right|_{x}$ and $\left.\varphi\right|_{x+l}$ as linear functionals on $\mathbb{R}^{n}$, this equation may be rewritten as

$$
\left.\varphi\right|_{x+l}=\left.\varphi\right|_{x}-e_{l} \text {. }
$$

2.19. Definition. Let $\varphi^{D}$ be the 1 -form on $\mathbb{R}^{n}$ given by $\varphi^{D}=-\sum_{j=1}^{m} r_{j} u_{j} d v_{j}$, and set $\nabla^{D}=d+2 \pi i \varphi^{D}$. Note that under the identification of the tangent space $T_{x} \mathbb{R}^{n}$ with $\mathbb{R}^{n}$, we have $\left.\varphi^{D}\right|_{x}=-e_{x}$ for $x \in \mathbb{R}^{n}$. Thus $\varphi^{D}$ trivially satisfies Equation (2.18) and hence defines a connection on $L$. This connection will be called the distinguished connection on $L$.

2.20. Proposition. The Chern class of the line bundle $L$ constructed in 2.7 is represented by the 2 -form $\Omega=$ $\sum_{j=1}^{m} r_{j} d u_{j} \wedge d v_{j}$ on $\mathcal{L} \backslash \mathbb{R}^{n}$.

Proof. The Chern class is represented by $-\frac{1}{2 \pi i} \kappa$, where $\kappa$ is the curvature form defined by a choice of connection on $L$. (The cohomology class of this form is independent of the choice of connection.) We use the distinguished connection $\nabla^{D}$. Pulling back to the trivial bundle over $\mathbb{R}^{n}$, the curvature form is given by $d\left(2 \pi i \varphi^{D}\right)=$ $-2 \pi i \sum_{j=1}^{m} r_{j} d u_{j} \wedge d v_{j}$. This form, being translation-invariant, descends to a 2-form on $M$ which is the curvature form of the distinguished connection $\nabla^{D}$ on $L$. The proposition now follows.

Thus for every 2-dimensional integer cohomology class $[\Omega]$, we have constructed a line bundle with Chern class $[\Omega]$. Thus all complex line bundles over the torus arise from the above construction.

2.21. DEFINITION.

(i) Denote by $\bar{\rho}$ both the involutive isometry $\bar{\rho}: \mathbb{R}^{n} \rightarrow \mathbb{R}^{n}$ given by $x \mapsto-x$ and the induced isometry of $M$. This isometry of $M$ is covered by the map $\rho$ of $L$ given by $[(x, z)] \mapsto[(-x, z)]$. We obtain an involution $\rho^{*}: \mathcal{E}(L) \rightarrow \mathcal{E}(L)$ given by $\rho^{*}(s)=\rho \circ s \circ \bar{\rho}$; in particular, in the notation of Equation (2.11), we have $\rho^{*}(\bar{h})(\bar{x})=[(x, h(-x))]$. We will say that a connection on $L$ is $\mathbb{Z}_{2}$-invariant if it is invariant under $\rho$, i.e., $\nabla_{\bar{\rho}_{*}(X)} \circ \rho^{*}=\rho^{*} \circ \nabla_{X}$ for all $X \in T M$. Expressing the connection (pulled back to the trivial bundle over $\mathbb{R}^{n}$ ) as in Definition 2.14 , we see that it is $\mathbb{Z}_{2}$-invariant if and only if the 1 -form $\varphi$ is invariant under the involution $\bar{\rho}$. We will also say that $\varphi$ is $\mathbb{Z}_{2}$-invariant in this case. Under the identification of each tangent space $T_{x}\left(\mathbb{R}^{n}\right)$ with $\mathbb{R}^{n}$, the $\mathbb{Z}_{2}$-invariance of $\varphi$ says that $\left.\varphi\right|_{-x}(-X)=\left.\varphi\right|_{x}(X)$; i.e., $\left.\varphi\right|_{-x}=-\left.\varphi\right|_{x}$.

(ii) By an abuse of language, we will say that a connection $\nabla$ on a line bundle $L$ over $M$ is a translation-invariant connection if its curvature is a translation-invariant 2-form. (The pullback of such a connection to the bundle $\mathbb{R}^{n} \times \mathbb{C} \rightarrow \mathbb{R}^{n}$ is translation-invariant in the sense that each translation of $\mathbb{R}^{n}$ is covered by a map of $\mathbb{R}^{n} \times \mathbb{C}$ to itself preserving the connection. However, in general the analogous statement does not hold for the bundle $L \rightarrow M$.) We remark that the translation-invariant forms on the torus are precisely the harmonic forms.

2.22. REMARK. Since the curvature form of any connection is cohomologous to that of $\nabla^{D}$, and since the translationinvariant forms are precisely the harmonic forms, it follows by Hodge theory that the translation-invariant connections all have the same curvature form. 
2.23. Definition. Two connections $\nabla_{1}$ and $\nabla_{2}$ on $L$ are said to be gauge equivalent if there exists a bundle automorphism $F: L \rightarrow L$ that intertwines the two connections: $F \circ \nabla_{2}=\nabla_{1} \circ F$.

2.24. REMARK. Every Hermitian connection on $L$ is of the form

$$
\nabla=\nabla^{D}+2 \pi i \nu
$$

where $\nu$ is a real-valued 1-form on $M$. By the Hodge decomposition, we may write

$$
\nu=\alpha+d g+d^{*} \mu
$$

where $\alpha$ is a harmonic 1-form, $g \in C^{\infty}(M)$, and $\mu$ is a smooth 2-form on $M$. The harmonic form $\alpha$ may be viewed as a linear functional on $\mathbb{R}^{n}$.

2.26. Notation. Let $\mathcal{L}^{\prime} \subset\left(\mathbb{R}^{n}\right)^{\prime}$ denote the dual lattice to $\mathcal{L} ; \mathcal{L}^{\prime}$ consists of linear functionals on $\mathbb{R}^{n}$ taking integer values on $\mathcal{L}$.

2.27. Proposition. We use the notation of Definitions 2.14, 2.19, and 2.21 and Remark 2.24.

(i) A connection $\nabla=\nabla^{D}+2 \pi i \nu$ as in Equation (2.25) is translation-invariant if and only if $d^{*} \mu=0$.

(ii) A translation-invariant connection is also $\mathbb{Z}_{2}$-invariant if and only if $g$ is $\mathbb{Z}_{2}$-invariant and $\alpha(\mathcal{L}) \subseteq \frac{1}{2} \mathbb{Z}$.

(iii) The gauge equivalence class of a translation-invariant connection $\nabla=\nabla^{D}+2 \pi i \nu=\nabla^{D}+2 \pi i(\alpha+d g)$ is independent of the function $g$, and depends only upon the coset of $\alpha$ in $\mathcal{L}^{\prime} \backslash\left(\mathbb{R}^{n}\right)^{\prime}$.

2.28. REMARK. Since gauge equivalent connections give rise to the same spectra, we are interested in connections only up to gauge equivalence; thus from now on we will use (iii) to assume that $g=0$. Moreover, since altering $\alpha$ by an element $\beta \in \mathcal{L}^{\prime}$ does not affect the gauge equivalence class of the connection, only the coset of $\alpha$ in $\mathcal{L}^{\prime} \backslash\left(\mathbb{R}^{n}\right)^{\prime}$ is important to us.

Proof.

(i) The curvature forms of $\nabla$ and $\nabla^{D}$ differ by $2 \pi i d \nu=2 \pi i d d^{*} \mu$. Thus, if $d^{*} \mu=0$, then $\nabla$ is translationinvariant, since $\nabla^{D}$ is. Conversely, suppose that $\nabla$ is translation-invariant. Then $d d^{*} \mu$ is translation-invariant, i.e., harmonic. By Hodge theory, this means that $d d^{*} \mu=0$, i.e., $d^{*} \mu$ is closed. But being both closed and coclosed, is it harmonic; since it is also exact, it must be zero.

(ii) The condition that $\nabla$ be $\mathbb{Z}_{2}$-invariant is that $\bar{\rho}^{*} \nu=\nu$. The involution $\bar{\rho}$ is an isometry, so it preserves the Hodge decomposition, so this is equivalent to $\bar{\rho}^{*} d g=d g$ and $\bar{\rho}^{*} \alpha=\alpha$.

(iii) The Hermitian bundle automorphism $F: L \rightarrow L$ given by $[(x, z)] \mapsto\left[\left(x, e^{2 \pi i g(x)} z\right)\right]$ permits the definition of a new Hermitian connection $\nabla^{\prime}$ on $L$ by $\nabla_{X}^{\prime} s=F^{-1} \circ \nabla_{X}(F \circ s)$; these connections are gauge equivalent, being intertwined by $F$. The two connections are easily seen to be related by $\nabla^{\prime}=\nabla+2 \pi i d g$. Similarly, the bundle automorphism $[(x, z)] \mapsto\left[\left(x, e^{2 \pi i \beta(x)} z\right)\right]$ of $L$ permits the definition of a new gauge-equivalent Hermitian connection given by $\nabla+2 \pi i \beta$.

2.29. Definition. The torus $\mathcal{L} \backslash \mathbb{R}^{n}$ has a flat Riemannian metric defined by the inner product $g$ on $\mathbb{R}^{n}$. Given a connection $\nabla: \mathcal{E}(L) \rightarrow \mathcal{E}\left(T^{*}(M) \otimes L\right)$ on $L$, we can thus define an associated Laplacian $\Delta: \mathcal{E}(L) \rightarrow \mathcal{E}(L)$ given by $\Delta=-\operatorname{trace}\left(\nabla^{2}\right)$. If $X_{1}, \ldots, X_{n}$ is an orthonormal frame field on $M$, then we can express the Laplacian as

$$
\Delta=-\sum_{j=1}^{n}\left(\nabla_{X_{j}} \circ \nabla_{X_{j}}-\nabla_{\nabla_{X_{j}}^{L C}\left(X_{j}\right)}\right)
$$

where $\nabla^{L C}$ is the Levi-Civita connection on the tangent bundle to $M$. Since $(M, g)$ is a flat torus, we may choose the vector fields $X_{j}$ to be translation-invariant vector fields (equivalently, coordinate vector fields), so that the second term in the summand vanishes.

2.31. Notation. For $\nabla=d+2 \pi i \varphi$ as in Definition 2.14, we will denote the associated Laplacian by $\Delta_{\varphi}$. We will use the shorthand notation $\Delta^{D}$ for $\Delta_{\varphi^{D}}$. 
2.32. REMARK.

(i) The connection $\nabla$, viewed as a connection on the trivial bundle $\mathbb{R}^{n} \times \mathbb{C}$ over $\mathbb{R}^{n}$, gives rise to a Laplace operator $\widetilde{\Delta}$ on $\mathcal{E}\left(\mathbb{R}^{n} \times \mathbb{C}\right)$ defined by the same expression (2.30), where $X_{1}, \ldots, X_{n}$ is any orthonormal frame field on $\mathbb{R}^{n}$. (Again the expression is independent of the choice of orthonormal frame field.) If each $X_{i}$ is chosen to be a translation-invariant vector field on $\mathbb{R}^{n}$, then Equation (2.15) shows that $\nabla_{X_{i}}$ commutes with the action of $\mathcal{L}$ on $\mathcal{E}\left(\mathbb{R}^{n} \times \mathbb{C}\right)$ defined in Equation (2.9). It follows that $\widetilde{\Delta}$ commutes with the action and thus induces an operator on $\mathcal{E}(L)$, namely the Laplacian defined in Definition 2.29.

(ii) If the connection $\nabla$ is $\mathbb{Z}_{2}$-invariant as in Definition 2.21 , then the Laplacian commutes with the involution $\rho^{*}$ of $\mathcal{E}(L)$. Equivalently, under the identification of sections of $L$ with complex-valued functions on $\mathbb{R}^{n}$ satisfying Equation (2.12), we have $\Delta(h \circ \bar{\rho})(x)=(\Delta h)(-x)$.

2.33. Proposition. Let $L$ be a complex line bundle over the flat torus $(M, g)$. In the notation of Definition 2.29 , we have

$$
\Delta_{\varphi}=-\operatorname{div} \circ \operatorname{grad}-4 \pi i \varphi \circ \operatorname{grad}+4 \pi^{2}|\varphi|^{2}-2 \pi i \operatorname{div}\left(\varphi^{\#}\right)
$$

where the "index-raising" map \# : $T^{*} M \rightarrow T M$ is the inverse of the duality isomorphism $b: T M \rightarrow T * M$ arising from the Riemannian metric $g$ on $\mathbb{R}^{n}$.

Proof. For $h$ a smooth, complex-valued function on $\mathbb{R}^{n}$ satisfying Equation (2.12) and for $X$ a tangent vector to $\mathbb{R}^{n}$, we have $\nabla_{X} h=d h(X)+2 \pi i \varphi(X) h=(X+2 \pi i \varphi(X)) h$. Thus

$$
\nabla_{X} \circ \nabla_{X}(h)=X^{2} h-4 \pi^{2} \varphi(X) \varphi(X) h+2 \pi i X(\varphi(X)) h+4 \pi i \varphi(X) X h .
$$

Let $\left\{X_{1}, \ldots, X_{n}\right\}$ be an orthonormal, translation-invariant frame field on $M$. We have $\sum_{j=1}^{n} X_{j}^{2}(h)=\operatorname{div} \operatorname{grad} h$, $\sum_{j=1}^{n} \varphi\left(X_{j}\right) \varphi\left(X_{j}\right)=|\varphi|^{2}, \sum_{j=1}^{n} X_{j}\left(\varphi\left(X_{j}\right)\right)=\operatorname{div}\left(\varphi^{\#}\right)$, and $\sum_{j=1}^{n} \varphi\left(X_{j}\right) X_{j}(h)=\varphi(\operatorname{grad} h)$. Thus the proposition follows from Equation (2.34).

2.35. Notation. Given $[\alpha] \in \mathcal{L}^{\prime} \backslash\left(\mathbb{R}^{n}\right)^{\prime}$ and $Q \in C^{\infty}(M)$ (viewed as a periodic function on $\mathbb{R}^{n}$ ), let $S p e c_{\alpha}(Q ; L)$ denote the spectrum of the Schrödinger operator $\Delta_{\varphi^{D}+\alpha}+Q$ acting on smooth sections of $L$. The correspondence that associates to each $[\alpha] \in \mathcal{L}^{\prime} \backslash\left(\mathbb{R}^{n}\right)^{\prime}$ the spectrum $\operatorname{Spec}_{\alpha}(Q ; L)$ will be called the L-Bloch spectrum of $Q$. Usually, the line bundle $L$ will be understood and we will use "Bloch spectrum" to mean $L$-Bloch spectrum.

We will write $\operatorname{Spec}(Q ; L)$ for $\operatorname{Spec}_{0}(Q ; L)$, the spectrum of the Laplacian associated with the distinguished connection.

2.36. Proposition. In the notation of 2.35, $\operatorname{Spec}_{\alpha}(Q ; L)$ coincides with the spectrum of the operator $\Delta^{D}+Q$ acting on the space of all smooth functions on $\mathbb{R}^{n}$ satisfying

$$
h(x+l)=e^{2 \pi i \alpha(l)} e^{2 \pi i e_{l}(x)} h(x)
$$

for all $x \in M, l \in \mathcal{L}$, where $e_{l}$ is given as in Notation 2.4 .

Proof. Let $L_{\alpha}$ be the bundle over $M$ given by the quotient of $\mathbb{R}^{n} \times \mathbb{C}$ by the equivalence relation $(x, z) \equiv$ $\left(x+l, e^{2 \pi i \alpha(l)} e^{2 \pi i e_{l}(x)} z\right)$ for $l \in \mathcal{L}$. Sections of $L_{\alpha}$ may be viewed as functions on $\mathbb{R}^{n}$ satisfying Equation (2.37). The automorphism of $\mathbb{R}^{n} \times \mathbb{C}$ given by $(x, z) \mapsto\left(x, e^{2 \pi i \alpha(x)} z\right)$ intertwines $\nabla^{D}+2 \pi i \alpha$ with $\nabla^{D}$ and induces an isomorphism from $L$ to $L_{\alpha}$. Thus it intertwines $\Delta_{\varphi^{D}+\alpha}$ with the operator $\Delta^{D}$ acting on the space of all smooth functions on $\mathbb{R}^{n}$ satisfying Equation (2.37).

2.38. REMARK. In the case of the trivial line bundle $M \times \mathbb{C}$, the distinguished connection is given by $\nabla=d$, (i.e., $\varphi^{D}=0$ ), the associated Laplacian $\Delta$ is the Euclidean Laplacian $\Delta$ defined by the Euclidean metric $h$, and $e_{l}=0$ for every $l \in \mathcal{L}$. Thus $\operatorname{Spec}_{\alpha}(Q)$ is the spectrum of $\Delta+Q$ acting on the space of smooth functions on $\mathbb{R}^{n}$ satisfying $f(x+l)=e^{2 \pi i \alpha(l)} f(x)$ for all $x \in \mathbb{R}^{n}$ and $l \in \mathcal{L}$. Hence the notion of Bloch spectrum in Definition 2.35 in the case of a trivial bundle agrees with the classical Bloch (or Floquet) spectrum in the literature. 


\section{C. Nilmanifold structure on the principal circle bundle.}

We continue to use the conventions of Notation 2.4.

Let $\pi: L \rightarrow M$ be a smooth Hermitian complex line bundle over $\mathrm{M}$. The unit sphere bundle $P \rightarrow M$ of $L \rightarrow M$ whose fiber $P_{x}$, above $x \in M$ is given by $P_{x}=\left\{\xi \in L_{x} \mid\langle\xi, \xi\rangle=1\right\}$, is a principal $S^{1}$-bundle. Conversely, given the principal circle bundle $P$, one recovers the original line bundle $L$ as the associated vector bundle given as follows: The circle $S^{1}=U(1)$ acts on $\mathbb{C}$ by unitary transformations $\left(t \in S^{1}\right.$ acts as multiplication by $\left.e^{2 \pi i t}\right)$. We thus obtain a left action of $S^{1}$ on $P \times \mathbb{C}$ given by $g \cdot(p, z)=\left(p \cdot g^{-1}, g \cdot z\right)$ for $g \in S^{1},(p, z) \in P \times \mathbb{C}$. We let $L$ be the orbit space of this action,

$$
L=P \times_{S^{1}} \mathbb{C}=S^{1} \backslash(P \times \mathbb{C}) .
$$

Then $L$ is a complex line bundle over $\mathcal{L} \backslash \mathbb{R}^{n}$.

The space $\mathcal{E}(L)$ of smooth sections of $L$ may be identified with the space of $S^{1}$-equivariant smooth maps from $P$ to the representation space $\mathbb{C}$ :

$$
C^{\infty}(P, \mathbb{C})^{S^{1}}=\left\{f \in C^{\infty}(P, \mathbb{C}) \mid f\left(p g^{-1}\right)=g f(p) \forall p \in P, \forall g \in U(1)\right\} .
$$

(Given $f \in C^{\infty}(P, \mathbb{C})^{S^{1}}$, define its associated section $\sigma \in \mathcal{E}(L)$ by $\sigma(x)=[p, f(p)]$ where $p$ is any element of $P$ for which $\pi(p)=x$ and where $[p, z]$ denotes the equivalence class of $(p, z)$ in $L^{\prime}=S^{1} \backslash(P \times \mathbb{C})$.)

Every principal circle bundle over a torus has the structure of a two-step nilmanifold, the quotient of a two-step nilpotent Lie group by a cocompact discrete subgroup. In what follows, we will first construct a principal circle bundle with the structure of such a nilmanifold. We will then construct an associated bundle $L^{\prime}$ and show that it coincides with the line bundle $L$ constructed in Subsection 2B.

\section{C.1. Principal bundles.}

Define a two-step nilpotent Lie group structure on $\mathbb{R}^{n+1}$ by setting

$$
(x, t)(y, s)=\left(x+y, s+t+\sum_{j=1}^{m} r_{j} u_{j}(x) v_{j}(y)\right)=\left(x+y, s+t+e_{x}(y)\right)
$$

for all $x, y \in \mathbb{R}^{n}$ and $t, s \in \mathbb{R}$. (See Notation 2.4.) Let $N$ denote $\mathbb{R}^{n+1}$ with this Lie group structure. The derived group (i.e., the subgroup generated by all $\eta_{1} \eta_{2} \eta_{1}^{-1} \eta_{2}^{-1}$ with $\left.\eta_{1}, \eta_{2} \in N\right)$ is given by $\{(0, t): t \in \mathbb{R}\}$ and is central in $N$. We will denote the derived group by $(0, \mathbb{R})$. It is easily checked that the center consists of elements $(z, u)$ such that $z$ is in the kernel of the bilinear form $\Omega$. In this paper, we are interested only in the case in which $r_{1}, \ldots, r_{m}$ are all nonzero, i.e., $\Omega$ is nondegenerate, in which case the derived group coincides with the center. Thus we will refer to the derived group as the center in what follows.

The lattice $\mathcal{L}$ in $\mathbb{R}^{n}$ extends to a cocompact discrete subgroup $\Gamma$ of $N$ given by

$$
\Gamma=\{(l, k): l \in \mathcal{L}, k \in \mathbb{Z}\} .
$$

Letting $\bar{N}=(0, \mathbb{Z}) \backslash N$, where $(0, \mathbb{Z})$ is the integer lattice in $(0, \mathbb{R})$, then $\bar{N}$ is a nilpotent Lie group with center isomorphic to $S^{1}$. Set $\bar{\Gamma}=(0, \mathbb{Z}) \backslash \Gamma \cong \mathcal{L}$ and

$$
P^{\prime}=\Gamma \backslash N=\bar{\Gamma} \backslash \bar{N} .
$$

Denote elements of $P^{\prime}$ by $\overline{(x, t)}$, with $(x, t) \in N$. The surjective group homomorphism $N \rightarrow \mathbb{R}^{n}$ given by $(x, t) \mapsto x$ induces a submersion

$$
\pi: P^{\prime} \rightarrow \mathcal{L} \backslash \mathbb{R}^{n}=M
$$

with fiber $S^{1}=(0, \mathbb{Z}) \backslash(0, \mathbb{R})$ : 


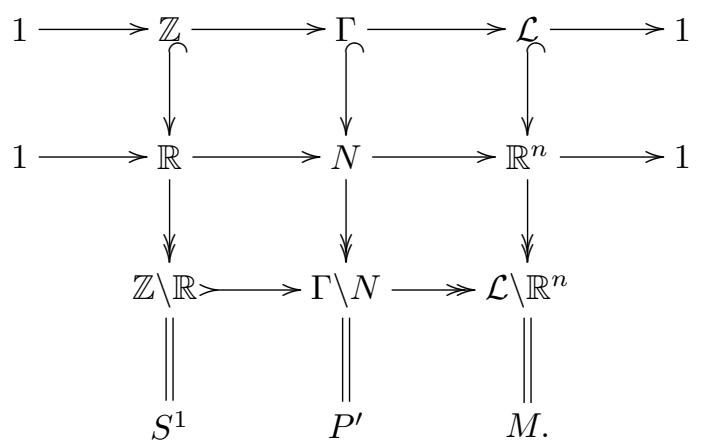
by

The action by translation of the center $(0, \mathbb{R})$ of $N$ on $N$ gives rise to a right (and left) action of $S^{1}$ on $P^{\prime}$ given

$$
\overline{(x, t)} \cdot e^{2 \pi i s}=\overline{(x, t+s)} .
$$

This action gives $P^{\prime}$ the structure of a principal circle bundle over $M$.

Let $L^{\prime}$ be the line bundle over $M$ associated with $P^{\prime}$ as in Equation (2.39). Elements of $L^{\prime}$ will be written $[\overline{(x, t)}, z]$. We have $[\overline{(x, t+s)}, z]=\left[\overline{(x, t)}, e^{2 \pi i s} z\right]$.

2.42. Notation. By Equation (2.40), sections of $L^{\prime}$ may be viewed as smooth functions $f: P^{\prime} \rightarrow \mathbb{C}$ satisfying $f(\overline{(x, t-s)})=e^{2 \pi i s} f(\overline{(x, t)})$. Such a function pulls back to a $\mathbb{C}$-valued function, which we again denote by $f$, on $\bar{N}$ satisfying the $S^{1}$-equivariance condition

$$
f(x, \overline{t-s})=e^{2 \pi i s} f(x, \bar{t})
$$

the latter in turn pulls back to a $\Gamma$-invariant function (still denoted $f$ ) on $N$ satisfying

$$
f(x, t-s)=e^{2 \pi i s} f(x, t)
$$

for all $s, t \in \mathbb{R}, x \in \mathbb{R}^{n}$ :

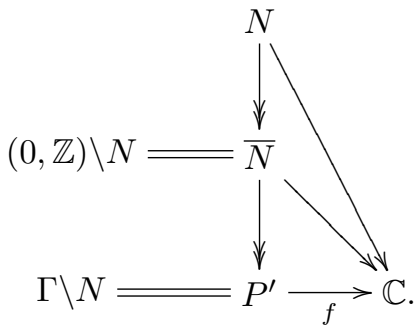

Note that the $\Gamma$-invariance of $f: N \rightarrow \mathbb{C}$ together with equation (2.43) says that

$$
f(x, t)=f((l, k)(x, t))=f\left(l+x, k+t+e_{l}(x)\right)=e^{-2 \pi i e_{l}(x)} f(l+x, t) .
$$

In order to compare the bundle $L^{\prime}$ with the bundle $L$ constructed in Subsection 2B, we consider the pullbacks of $P^{\prime}$ and $L^{\prime}$ to bundles over $\mathbb{R}^{n}$. The former is simply $\bar{N}$, with $S^{1}$ action given by $(x, \bar{t}) \cdot e^{2 \pi i s}=(x, \overline{t+s})$. The latter is the bundle $\widetilde{L^{\prime}}=\bar{N} \times_{S^{1}} \mathbb{C}$ over $\mathbb{R}^{n}$ given by equivalence classes $[(x, \bar{t}), z]$ with $[(x, \bar{t}), z]=\left[(x, \overline{t-s}), e^{2 \pi i s} z\right]$. In particular, $[(x, \bar{t}), z]=\left[(x, \overline{0}), e^{2 \pi i t} z\right]$.

2.45. Proposition. In the notation above, define $T: \widetilde{L}^{\prime} \rightarrow \mathbb{R}^{n} \times \mathbb{C}$ by $T([(x, \bar{t}), z])=\left(x, e^{2 \pi i t} z\right)$. Then $T$ induces an equivalence, also denoted $T$, between the line bundle $L^{\prime}$ over $M$ constructed above and the line bundle $L$ constructed in Definition 2.7. Under this equivalence, a section $f$ of $L^{\prime}$, viewed as a function on $N$ satisfying Equation (2.43), corresponds to the section $h$ of $L$ given by $h(x)=f(x, 0)$, where $h$ is as in Equation (2.11). 
Proof. The group $\bar{\Gamma} \cong \mathcal{L}$ acts on the line bundle $\widetilde{L}^{\prime}$ by

$$
(l, \overline{0}) \cdot[(x, \bar{t}), z]=[(l, \overline{0})(x, \bar{t}), z]=\left[\left(l+x, \bar{t}+\overline{e_{l}(x)}\right), z\right]=\left[(l+x, \bar{t}), e^{2 \pi i e_{l}(x)} z\right] .
$$

The bundle $L^{\prime}$ is the quotient of $\widetilde{L}^{\prime}$ by this group action. The map $T$ carries orbits of the action of $\bar{\Gamma}$ on $\widetilde{L}^{\prime}$ to equivalence classes of $\mathbb{R}^{n} \times \mathbb{C}$ with respect to the equivalence relation in Equation (2.8). The proposition follows.

2C.2. Connections on the principal bundle.

The coordinate vector field $\frac{\partial}{\partial t}$ on $\mathbb{R}^{n+1}$ with respect to the global coordinates $(x, t)$ is invariant under left translations by elements of $\Gamma$ and thus induces a vector field, which we still denote by $\frac{\partial}{\partial t}$, on $P^{\prime}$. This vector field is the infinitesimal generator of the $S^{1}$ action on $P^{\prime}$. A connection on $P^{\prime}$ is specified by a real-valued 1-form $\omega$ on $P^{\prime}$ which is invariant under the $S^{1}$ action and satisfies $\omega\left(\frac{\partial}{\partial t}\right)=1$. (Here we are using the canonical identification of the Lie algebra of the Lie group $S^{1}$ with $\mathbb{R}$.) The kernel $\mathcal{H}$ of $\omega$ is referred to as the horizontal distribution defined by the connection. We have

$$
\mathcal{H}=\left\{X-\omega(X) \frac{\partial}{\partial t}: X \in T P^{\prime}\right\}
$$

and $\pi_{* p}$ carries $\mathcal{H}_{p}$ isomorphically to $T_{\pi(p)}(M)$ for all $p \in P^{\prime}$. The horizontal distribution $\mathcal{H}$ is invariant under the action of $S^{1}$. Conversely, any $S^{1}$-invariant $n$-plane distribution on $P^{\prime}$, complementary at each point to $\frac{\partial}{\partial t}$, defines a connection on $P^{\prime}$.

A connection specified by a 1-form $\omega$ on $P^{\prime}$ defines a connection $\nabla$ on the associated line bundle $L^{\prime}$ as follows. Given $x \in M$ and $X \in T_{x}(M)$, choose $p \in \pi^{-1}(x)$ and let $\widetilde{X}$ denote the unique horizontal lift of $X$ in $T_{p}\left(P^{\prime}\right)$; i.e., $\widetilde{X} \in \mathcal{H}_{p}$ and $\pi_{*}(\widetilde{X})=X$. Given a section of $L^{\prime}$, viewed as an element of $C^{\infty}\left(P^{\prime}, \mathbb{C}\right)^{S^{1}}$ (i.e., as an $S_{1}$ equivariant smooth map $P^{\prime} \rightarrow \mathbb{C}$ ), we then define $\nabla_{X}(f)=\tilde{X}(f)$. (The fact that $\mathcal{H}$ is $S^{1}$-invariant guarantees that this definition is independent of the choice of $p$.)

2.47. EXAmple. Let $e_{1}, \ldots, e_{n}$ denote the standard basis vectors of $\mathbb{R}^{n}$. The left-invariant vector field $\widetilde{X}_{j}$ on $N$ whose value at the identity is given by $\frac{\partial}{\partial x_{j}}$ is given at $(x, t)$ by

$$
\widetilde{X}_{j}=\left.\frac{d}{d s}\right|_{s=0}(x, t)\left(s e_{j}, 0\right)=\left.\frac{d}{d s}\right|_{s=0}\left(x+s e_{j}, t+s e_{x}\left(e_{j}\right)\right)=\frac{\partial}{\partial x_{j}}+e_{x}\left(e_{j}\right) \frac{\partial}{\partial t} .
$$

A left-invariant vector field $\widetilde{X}$ on $N$ induces a vector field, also denoted $\widetilde{X}$, on $P^{\prime}$. We will abuse terminology and refer to $\widetilde{X}$ as a left-invariant vector field on $P^{\prime}$. Let $\mathcal{H}$ be the distribution on $P^{\prime}$ spanned by the left-invariant vector fields $\widetilde{X}_{1}, \ldots, \widetilde{X}_{n}$ given in equation (2.48). Viewing sections of $L^{\prime}$ as $\Gamma$-invariant functions $f$ on $N$ satisfying Equation (2.43), then $\frac{\partial f}{\partial t}=-2 \pi i f$. Thus comparing Equation (2.48) and Definition 2.19, we see that the connection on $L^{\prime}$ defined by $\mathcal{H}$ corresponds to the distinguished connection on $L$ under the equivalence between $L$ and $L^{\prime}$ given in Proposition 2.45.

More generally, for $\alpha=\sum_{j=1}^{n} a_{j} d x_{j}$ a harmonic 1-form on $M$, the translation-invariant connection $\nabla=$ $d+2 \pi i\left(\varphi^{D}+\alpha\right)$ on $L$ corresponds to the connection on $L^{\prime}$ for which the horizontal distribution (on $\left.P^{\prime}\right)$ is spanned by the left-invariant vector fields $\widetilde{X}_{j}-a_{j} \frac{\partial}{\partial t}, j=1, \ldots, n$. (Note that $\frac{\partial}{\partial t}$ is a left-invariant vector field.) Thus by Proposition 2.27, a connection on $L^{\prime}$ corresponds to a translation-invariant connection on $L$ if and only if its associated horizontal distribution $\mathcal{H}$ is spanned by left-invariant vector fields, or equivalently, if the one-form $\omega$ on $P^{\prime}$ lifts to a left-invariant one-form on $N$.

Suppose that $\nabla$ is a translation-invariant connection so that $\mathcal{H}$ is spanned by left-invariant vector fields. The Riemannian inner product $h$ on $M$ gives rise to an inner product on $\mathcal{H}$. Let $\widetilde{X}_{1}, \ldots, \widetilde{X}_{n}$ denote an orthonormal basis of $\mathcal{H}$. Then the Laplacian on $L^{\prime}$ associated to the connection $\nabla$ is given by

$$
\Delta(f)=-\sum_{j=1}^{n} \widetilde{X}_{j}^{2}(f)
$$


where, as usual, we view $f$ as a $\Gamma$-invariant function on $N$ satisfying equation (2.43).

\section{INVERSE SPECTRAL RESULTS ON RECTANGULAR TORI.}

3.1. NOTATION.

(i) Let $n=2 m$ be an even integer and let $(u, v)=\left(u_{1}, \ldots, u_{m}, v_{1}, \ldots, v_{m}\right)$ be the standard coordinates on $\mathbb{R}^{n}$. Given $m$-tuples $a=\left(a_{1}, \ldots, a_{m}\right)$ and $b=\left(b_{1}, \ldots, b_{m}\right)$ of positive real numbers, give $\mathbb{R}^{n}$ the inner product for which the standard ordered basis elements are orthogonal and have lengths $a_{1}, \ldots, a_{m}, b_{1}, \ldots, b_{m}$, respectively. Let $M=M_{a, b}$ be the rectangular torus $\mathbb{Z}^{n} \backslash \mathbb{R}^{n}$ with the metric defined by this inner product.

(ii) Given an $m$-tuple $r=\left(r_{1}, \ldots, r_{m}\right)$ of positive integers such that $r_{1}\left|r_{2}\right| \ldots \mid r_{m}$, let $\Omega_{r}=\sum_{j=1}^{m} r_{j} d u_{j} \wedge d v_{j}$. The construction in Section 2 gives us a line bundle over $M$ with Chern class represented by $\Omega_{r}$; we denote this bundle by $L_{r}$. We will work in the setting of Subsection 2C, viewing the principal circle bundle given by the unit vectors in $L_{r}$ as a nilmanifold. We will write elements of the nilpotent group $N$ in the form $(u, v, t)$, with $u, v \in \mathbb{R}^{m}, t \in \mathbb{R}$. Comparing with the notation in Subsection $2 \mathrm{C}$, an element $(x, t) \in N$ in the notation of Subsection $2 \mathrm{C}$ will be written as $(u(x), v(x), t)$, where $u(x)$ and $v(x)$ are defined as in Notation 2.4(i). The group multiplication in $N$ is given by

$$
(u, v, t)\left(u^{\prime}, v^{\prime}, t^{\prime}\right)=\left(u+u^{\prime}, v+v^{\prime}, t+t^{\prime}+\sum_{j=1}^{m} r_{j} u_{j} v_{j}^{\prime}\right) .
$$

The discrete group $\Gamma$ is given by

$$
\Gamma=\left\{(u, v, t) \in N: u, v \in \mathbb{Z}^{m}, t \in \mathbb{Z}\right\} .
$$

Thus under the identifications discussed in Subsection 2C, sections of $L_{r}$ will be viewed as $\Gamma$-invariant functions on $N$ satisfying $f(u, v, t+s)=e^{-2 \pi i s} f(u, v, t)$ (see Equation (2.43)).

3.3. REMARK. Recall Remark 2.6. Since in 3.1, we fixed the basis used to construct $\Omega_{r}$ and the associated line bundle $L_{r}$, the bundles $L_{r}$ do not represent all possible line bundles over $M$. However, in the case of twodimensional tori (so $m=1$ and we drop the subscripts on $u, v$ ), the bundles $L_{r}$, as $r$ varies over the positive integers, range over all equivalence classes of line bundles with positive Chern number. As in Subsections $2 \mathrm{~B}$ and $2 \mathrm{C}$, all the results below continue to make sense if we allow $r$ to be negative, so as to obtain all nontrivial line bundles over $M$ in the two-dimensional case.

\subsection{NOTATION.}

(i) Given $m$-tuples $a, b$, and $r$ as in 3.1, define an operator $D_{r, a, b}$ on $C^{\infty}\left(\mathbb{R}^{m}\right)$ by

$$
D_{r, a, b}=\sum_{j=1}^{m}\left(\left(2 \pi \frac{r_{j}}{b_{j}} s_{j}\right)^{2}-\frac{1}{a_{j}^{2}} \frac{\partial^{2}}{\partial s_{j}^{2}}\right)
$$

where $s_{1}, \ldots, s_{m}$ are the standard coordinates on $\mathbb{R}^{m}$.

Under the change of coordinates on $\mathbb{R}^{m}$ given by setting $w_{j}=\sqrt{\frac{a_{j}}{b_{j}}} s_{j}$, the operator $D_{r, a, b}$ transforms to the operator

$$
\widetilde{D}_{r, a, b}=\sum_{j=1}^{m} \frac{1}{a_{j} b_{j}}\left(\left(2 \pi r_{j} w_{j}\right)^{2}-\frac{\partial^{2}}{\partial w_{j}^{2}}\right) .
$$

In particular, the operator depends only on the products $a_{j} b_{j}$, not on $a_{j}$ and $b_{j}$ individually.

(ii) Suppose $q \in C^{\infty}\left(\mathbb{R}^{m}\right)$ is periodic with respect to $\mathbb{Z}^{m}$. For $c=\left(c_{1}, \ldots, c_{m}\right) \in \mathbb{Z}^{m}$, define $q_{r, c} \in C^{\infty}\left(\mathbb{R}^{m}\right)$ by

$$
q_{r, c}(u)=q\left(u_{1}-\frac{c_{1}}{r_{1}}, \ldots, u_{m}-\frac{c_{m}}{r_{m}}\right) .
$$

Since $q$ is periodic, $q_{r, c}$ depends only on the coset of $c$ in $\left(r_{1} \mathbb{Z} \times \cdots \times r_{m} \mathbb{Z}\right) \backslash \mathbb{Z}^{m}$. Let $S(q, r, a, b)$ denote the join of the spectra of the operators $D_{r, a, b}+q_{r, c}$ as $c$ varies over the right coset space $\left(r_{1} \mathbb{Z} \times \cdots \times r_{m} \mathbb{Z}\right) \backslash \mathbb{Z}^{m}$. 
3.5. THEOREM. Let $a, b$ and $r$ be as in Notation 3.1, and let $M=M_{a, b}$ and $L_{r}$ be the corresponding rectangular torus and line bundle as in 3.1. Let $q \in C^{\infty}\left(\mathbb{R}^{m}\right)$ be periodic with respect to $\mathbb{Z}^{m}$.

(1) Define $Q \in C^{\infty}(M)$ by $Q(u, v)=q(u)$. Then the spectrum of the Schrödinger operator $\Delta+Q$ acting on $\mathcal{E}\left(L_{r}\right)$ coincides with $S(q, r, a, b)$ as defined in Notation 3.4.

(2) Define $Q \in C^{\infty}(M)$ by $Q(u, v)=q(v)$. Then the spectrum of the Schrödinger operator $\Delta+Q$ acting on $\mathcal{E}\left(L_{r}\right)$ coincides with $S(q, r, b, a)$.

Before proving the theorem, we give several applications.

3.6. COROllary. Let $M$ be a rectangular 2-dimensional torus for which the side lengths a and $b$ are distinct positive integers. Let $L$ be any line bundle over $M$ with nondegenerate Chern class. Then there exist pairs of noncongruent potentials on $M$ which are isospectral with respect to the Schrödinger operator defined by the distinguished translation-invariant connection on $L$.

Proof. By Remark 3.3, it suffices to consider the line bundles $L_{r}$ given in Notation 3.1, where we allow $r$ to be an arbitrary nonzero integer. Fix $r$. Let $q \in C^{\infty}(\mathbb{R})$ be periodic with period $\frac{1}{a r}$, and let $\widetilde{q}(s)=q\left(\frac{b}{a} s\right)$. Define $Q_{1}(u, v)=q(u)$ and $Q_{2}(u, v)=\widetilde{q}(v)$. We show that $Q_{1}$ and $Q_{2}$ are isospectal potentials for the Schrödinger operator on $L_{r}$. In the notation of 3.4(ii), we have that $q_{r, c}=q$ and $\widetilde{q}_{r, c}=\widetilde{q}$ for all $c \in \mathbb{Z}$ due to the periodicity condition on $q$. By Theorem 3.5, the spectrum of $\Delta+Q_{1}$ is given by $S(q, r, a, b)$, which is equal to the join of $r$ copies of the spectrum of $D_{r, a, b}+q$. By the change of variables in Notation 3.4(ii), the spectrum of $\Delta+Q_{1}$ is thus equal to the join of $r$ copies of the spectrum of $\widetilde{D}_{r, a, b}+g$ where $g(s)=q\left(\sqrt{\frac{b}{a}} s\right)$. Again by Theorem 3.5 , the spectrum of $\Delta+Q_{2}$ is equal to $S(\widetilde{q}, r, b, a)$, which is in turn equal to the join of $r$ copies of the spectrum of $D_{r, b, a}+\widetilde{q}$. By the change of variables $u=\sqrt{\frac{b}{a}} s$, the operator $D_{r, b, a}+\widetilde{q}$ is carried to $\widetilde{D}_{r, a, b}+g$, since $\widetilde{q}\left(\sqrt{\frac{a}{b}} u\right)=q\left(\frac{b}{a} \sqrt{\frac{a}{b}} u\right)=q\left(\sqrt{\frac{b}{a}} u\right)=g(u)$. Since $a \neq b$, it is clear that the potentials $Q_{1}$ and $Q_{2}$ are not congruent. The theorem follows.

3.7. REMARK. For $2 m$-dimensional rectangular tori given as in Notation 3.1 with $\frac{a_{j}}{b_{j}}$ rational and not equal to one for all $j$, a similar proof shows that for each $r=\left(r_{1}, \ldots, r_{m}\right)$, there exist pairs of noncongruent potentials on $M$ which are isospectral with respect to the Schrödinger operator defined by the distinguished translation-invariant connection on $L_{r}$.

3.8. Definition. By the spectrum of a line bundle $L$ over a flat torus $M$, we will mean the spectrum of the Laplace operator associated with the distinguished translation-invariant connection on $L$.

3.9. COROLlaRY. For every even integer $n \geq 4$, there exist an $n$-dimensional rectangular flat torus $M$ and $a$ pair of isospectral, topologically distinct, line bundles over M. The Chern classes of these bundles have different invariant factors.

Proof. We illustrate the Corollary with a four-dimensional example. Choose $a$ so that $a_{1}=a_{2}$ and let $b=(1,2)$. Set $r=(1,4)$ and $r^{\prime}=(2,2)$. Since $\frac{r_{1}}{b_{1}}=\frac{r_{2}^{\prime}}{b_{2}}$ and $\frac{r_{2}}{b_{2}}=\frac{r_{1}^{\prime}}{b_{1}}$, we see from Theorem 3.5 (with $Q=0$ ) and from the definition of $D_{r, a, b}$ in Notation 3.4 that the line bundles $L_{r}$ and $L_{r^{\prime}}$ over $M$ are isospectral.

3.10. REMARK. In the setting of Corollary 3.9, there is also a large family of potential functions $Q$ on $M$ so that, for each $Q$, the Schrödinger operators $\Delta+Q$ acting on sections of the two distinct bundles have the same spectrum.

3.11. COROLLARY. In every even dimension $2 m, m \geq 1$, there exist pairs of nonisometric rectangular tori $M$ and $M^{\prime}$ such that for every choice of $r=\left(r_{1}, \ldots, r_{m}\right)$ as in Notation 3.1, the corresponding line bundles $L_{r}$ and $L_{r}^{\prime}$ over $M$ and $M^{\prime}$, respectively, have the same spectrum in the sense of Corollary 3.9. In the case of two-dimensional rectangular tori $M$, the spectrum of a nontrivial line bundle over $M$ depends only on the area of the torus and the Chern number of the bundle. 
Proof. We use the notation of 3.1. Choose $m$-tuples $a, b, a^{\prime}$ and $b^{\prime}$ of positive real numbers in such a way that $a_{j} b_{j}=a_{j}^{\prime} b_{j}^{\prime}$ for every $j=1, \ldots, m$. Let $M=M_{a, b}$ and $M^{\prime}=M_{a^{\prime}, b^{\prime}}$. Choose $r$ arbitrarily subject only to the condition $r_{1}|\ldots| r_{m}$ and let $L_{r}$ and $L_{r}^{\prime}$ be the corresponding line bundles over $M$ and $M^{\prime}$ respectively. From the expression $\widetilde{D}_{r, a, b}$ in Notation 3.4(ii) and by Theorem 3.5, with $Q=0$, we see that $L_{r}$ and $L_{r}^{\prime}$ have the same spectrum. In particular, in the two-dimensional case, (writing $a=a_{1}$ and $b=b_{1}$ ), the spectrum of the line bundle $L_{r}$ over $M$ depends only on $a b$ and $r$. Since $a b$ is the area of the torus and $r$ the Chern number of the line bundle, the final statement of the corollary follows. (Here we allow $r$ to take on negative as well as positive values; see Remark 3.3.)

We now turn to the proof of Theorem 3.5.

\section{Proof.}

(1) We use the notation of 3.1. As in Notation 2.42, smooth sections of the line bundle $L_{r}$ may be identified with elements of the space of $\Gamma$-periodic complex-valued smooth functions on $N$ satisfying

$$
f(u, v, t)=e^{-2 \pi i t} f(u, v, 0) .
$$

Let $U_{j}$, respectively $V_{j}$, denote the left-invariant vector field on $N$ whose value at the identity is $\frac{\partial}{\partial u_{j}}$, respectively, $\frac{\partial}{\partial v_{j}}$. By Equation (2.49), the Laplacian $\Delta$ with respect to the distinguished connection on $L_{r}$ is given by

$$
\Delta=-\sum_{j=1}^{m}\left(\frac{1}{a_{j}^{2}} U_{j}^{2}+\frac{1}{b_{j}^{2}} V_{j}^{2}\right) .
$$

The integral curve of $U_{j}$ (respectively, $V_{j}$ ) through the identity element of $N$ is given by $\exp \left(s U_{j}\right)=\left(s e_{j}, 0,0\right)$ (respectively, $\exp \left(s V_{j}\right)=\left(0, s e_{j}, 0\right)$ ), where $e_{j}$ is the $j$ th standard basis vector of $\mathbb{R}^{m}$.

Left-invariant vector fields are defined as derivatives of right translations (i.e., $X f(p)=\left.\frac{d}{d t}\right|_{t=0} f(p \exp (t X))$ for $p \in N$ ), so we can understand the action of the Laplacian on the smooth sections of $L_{r}$ by analyzing the right action of $N$ on the larger space $\mathcal{K}$ of square integrable sections. As will be explained below, it is well known that this right action is unitarily equivalent to the direct sum of $r_{1} \cdots r_{m}$ copies of the action $\tau$ of $N$ on $L^{2}\left(\mathbb{R}^{m}\right)$ given by

$$
(\tau(u, v, t) g)(w)=e^{-2 \pi i\left(t+\sum_{j=1}^{m} r_{j} w_{j} v_{j}\right)} g(w+u) .
$$

This action of $N$ on $L^{2}\left(\mathbb{R}^{m}\right)$ gives rise to an infinitesimal action of the Lie algebra $\mathfrak{n}$ of $N$; this action satisfies $\tau_{*}\left(U_{j}\right) g=\frac{\partial g}{\partial w_{j}}$ and $\tau_{*}\left(V_{j}\right) g=-2 \pi i r_{j} w_{j} g$. Thus, under this equivalence, the Laplacian is carried to the operator $D_{r, a, b}$ defined in Notation 3.4.

We will make this equivalence precise in order to see its effect on the Schrödinger operator $\Delta+Q$. The presentation below of this equivalence was shown to the first author by E. N. Wilson during preparation of the article [GW].

In order to avoid cumbersome notation, we will carry out the proof in the case $m=1$. For the general case, the reader may simply replace $r \alpha v$ by $\sum_{j=1}^{m} r_{j} \alpha_{j} v_{j}$, replace $c+r \alpha$ by $\left(c_{1}+r_{1} \alpha_{1}, \ldots, c_{m}+r_{m} \alpha_{m}\right)$, replace $\mathbb{Z}$ by $\mathbb{Z}^{m}$, etc.

Let $f \in \mathcal{K}$. By Equation (3.12) and $\Gamma$-periodicity, we have

$$
f(u, v, t)=f(u+\alpha, v+\beta, t+r \alpha v)=e^{-2 \pi i r \alpha v} f(u+\alpha, v+\beta, t)
$$

for all $\alpha, \beta \in \mathbb{Z}$. In particular, $f$ is periodic with respect to $\mathbb{Z}$ in $v$ and we may perform a Fourier decomposition in the $v$ variables:

$$
f(u, v, t)=\sum_{c \in \mathbb{Z}} \hat{f}_{c}(u) e^{-2 \pi i c v} e^{-2 \pi i t} .
$$

(We are writing $\hat{f}_{c}$ rather than $\hat{f}_{-c}$ here for notational convenience.)

By Equation (3.14), we see that

$$
\hat{f}_{c+r \alpha}(u)=\hat{f}_{c}(u+\alpha)
$$


for all $\alpha, \in \mathbb{Z}$. Thus choosing a representative $c$ of each coset $\bar{c}$ of $r \mathbb{Z}$ in $\mathbb{Z}$, we may write

$$
f(u, v, t)=\sum_{\bar{c} \in r \mathbb{Z} \backslash \mathbb{Z}} f_{\bar{c}}(u, v, t)
$$

where

$$
f_{\bar{c}}(u, v, t)=e^{-2 \pi i t} \sum_{\alpha \in \mathbb{Z}} \hat{f}_{c}(u+\alpha) e^{-2 \pi i(c+r \alpha) v} .
$$

Each of the functions $f_{\bar{c}}$ on $N$ is $\Gamma$-invariant. Thus we obtain a decomposition

$$
\mathcal{K}=\bigoplus_{\bar{c} \in r \mathbb{Z} \backslash \mathbb{Z}} \mathcal{K}_{\bar{c}}
$$

where $\mathcal{K}_{\bar{c}}=\left\{f_{\bar{c}}: f \in \mathcal{K}\right\}$. (In the case of a $2 m$-dimensional torus, $r \mathbb{Z} \backslash \mathbb{Z}$ is replaced by $\left(r_{1} \mathbb{Z} \times \cdots \times r_{m} \mathbb{Z}\right) \backslash \mathbb{Z}^{m}$. In particular, the index set in the right hand side of Equation (3.16) has order $r_{1} \ldots r_{m}$.)

For $f \in \mathcal{K}$, the square of the $L^{2}$ norm of $f$ as a section of the bundle $L_{r}$ is given by

$$
a b \sum_{c \in \mathbb{Z}} \int_{0}^{1}\left|\hat{f}_{c}\right|^{2}(x) d x=a b \sum_{\bar{c} \in r \mathbb{Z} \backslash \mathbb{Z}} \int_{\mathbb{R}}\left|\hat{f}_{\bar{c}}\right|^{2}(x) d x .
$$

Thus for each coset $\bar{c}$, we obtain a unitary isomorphism $T_{\bar{c}}: \mathcal{K}_{\bar{c}} \rightarrow L^{2}(\mathbb{R})$ given by

$$
T_{\bar{c}}(f)=\sqrt{a b} \hat{f}_{c}
$$

where $c$ is an arbitrary but fixed choice of coset representative.

Let $\rho$ denote the right action of $N$ on $\mathcal{K}$. We have

$$
\left(\rho\left(u^{\prime}, v^{\prime}, t^{\prime}\right) f\right)(u, v, t)=f\left(u+u^{\prime}, v+v^{\prime}, t+t^{\prime}+r u v^{\prime}\right)
$$

and thus the Fourier coefficients of $\rho\left(u^{\prime}, v^{\prime}, t^{\prime}\right) f$ are given by

$$
\left(\rho\left(u^{\prime}, v^{\prime}, t^{\prime}\right) f\right)_{c}=e^{-2 \pi i\left[t^{\prime}+(r u+c) v^{\prime}\right]} \hat{f}_{c}\left(u+u^{\prime}\right) .
$$

In particular, the right action leaves each subspace $\mathcal{K}_{\bar{c}}$-invariant. Moreover, $T_{\bar{c}}$ intertwines the right action $\rho$ on $\mathcal{K}_{\bar{c}}$ with the action $\sigma$ of $N$ on $L^{2}(\mathbb{R})$ given by $\sigma\left(u^{\prime}, v^{\prime}, t^{\prime}\right) g(w)=e^{-2 \pi i\left[t^{\prime}+(r w+c) v^{\prime}\right]} g\left(w+u^{\prime}\right)$. For each $c$ as above, define $S_{\bar{c}}: L^{2}(\mathbb{R}) \rightarrow L^{2}(\mathbb{R})$ by

$$
S_{\bar{c}}(g)(w)=g_{r, c}(w)=g\left(w-\frac{c}{r}\right) .
$$

Then $S_{\bar{c}} \circ T_{\bar{c}}$ intertwines the the right action $\rho$ on $\mathcal{K}_{\bar{c}}$ with the action $\tau$ given in Equation (3.13) and carries the Laplacian $\Delta$ on $\mathcal{K}_{\bar{c}}$ to the operator $D_{r, a, b}$ on $L^{2}(\mathbb{R})$.

Note that multiplication by the potential function $Q$ (given as in part (i) of the theorem by $Q(u, v)=q(u)$ ) carries $\mathcal{K}$ to itself, leaves each $\mathcal{K}_{\bar{c}}$ invariant, and satisfies, for $f \in \mathcal{K},(Q f)_{\bar{c}}(u)=q(u) \hat{f}_{c}(u)$. Hence $T_{\bar{c}}(Q f)=$ $q T_{\bar{c}} f$. We thus have $S_{\bar{c}} \circ T_{\bar{c}}(Q f)=q_{r, c} S_{\bar{c}} \circ T_{\bar{c}}(f)$. It follows that $S_{\bar{c}} \circ T_{\bar{c}}$ intertwines the Schrödinger operator $\Delta+Q$ on $\mathcal{K}_{\bar{c}}$ with the operator $D_{r, a, b}+q_{r, c}$ on $L^{2}(\mathbb{R})$. This completes the proof of (1).

(2) The asymmetry between the roles of $u$ and $v$ above is due to our choice of coordinates on $N$. We have been denoting elements of $N$ as $(u, v, t)$. To avoid confusion as we introduce new coordinates, we will temporarily write $(u, v, t)_{1}$ for $(u, v, t)$. Define new coordinates by setting $(u, v, t)_{2}=(u, v,-t+r u v)_{1}$. An elementary computation using Equation (3.2) shows that

$$
(u, v, t)_{2}\left(u^{\prime}, v^{\prime}, t^{\prime}\right)_{2}=\left(u+u^{\prime}, v+v^{\prime}, t+t^{\prime}+r v u^{\prime}\right)_{2}
$$

Observe that the discrete subgroup $\Gamma$ of $N$ given by all elements with integer coordinates $(u, v, t)_{1}$ still consists precisely of the points whose coordinates $(u, v, t)_{2}$ are integers. However, in the new coordinates, we have for $\alpha, \beta \in \mathbb{Z}$

$$
(\alpha, 0,0)_{2}(u, v, t)_{2}=(u+\alpha, v, t)_{2}
$$

and

$$
(0, \beta, 0)_{2}(u, v, t)_{2}=(u, v+\beta, t+r \beta u)_{2} .
$$


Thus the left $\Gamma$-invariant functions are periodic in $u$ rather than in $v$. The integral curves of $U$ and $V$ through the identity element of $N$ are given by exactly the same expression in the new coordinates as in the old.

The function space $\mathcal{K}$ is given in our new coordinates by those left $\Gamma$-invariant functions on $N$ satisfying $f\left((u, v, t)_{2}\right)=e^{2 \pi i t} f\left((u, v, 0)_{2}\right)$. Write $\overline{\mathcal{K}}=\{\bar{f}: f \in \mathcal{K}\}$, where $\bar{f}$ denotes the complex conjugate, and define $B: \mathcal{K} \rightarrow \overline{\mathcal{K}}$ by $f \mapsto \bar{f}$. Note that the actions of the Schrödinger operator $\Delta+Q=-\left(\frac{1}{a^{2}} U^{2}+\frac{1}{b^{2}} V^{2}\right)+Q$ on $\mathcal{K}$ and $\overline{\mathcal{K}}$ are intertwined by $B$ (recall that $Q$ is real-valued). Thus we may consider $\overline{\mathcal{K}}$ rather than $\mathcal{K}$. Since $\overline{\mathcal{K}}$ consists of $\Gamma$-invariant functions satisfying $f\left((u, v, t)_{2}\right)=e^{-2 \pi i t} f\left((u, v, 0)_{2}\right)$, the computation of the spectrum is now identical to that carried out in part (i) except that the roles of $u$ and $v$ are switched. Thus (ii) follows.

\section{TORI WITH NONDEGENERATE LENGTH SPECTRUM.}

In this section we prove positive inverse spectral results, including Theorem 1.3, Theorem 1.4, and additional results concerning the odd part of the potential.

Let $L$ be a line bundle over the torus $M=\mathcal{L} \backslash \mathbb{R}^{2 m}$ for which all the invariant factors $r_{1}, \ldots, r_{m}$ of the Chern class are equal to one. Let $\Omega$ be the translation-invariant 2-form representing the Chern class of $L$. We may view $\Omega$ as a bilinear form on $\mathbb{R}^{2 m}$. Recall that $\Omega(x, y)=e_{x}(y)-e_{y}(x)$ (see Notation 2.4). Let $\mathcal{L}^{\prime}$ be the lattice in $\mathbb{R}^{2 m}$ dual to $\mathcal{L}$, i.e., $\mathcal{L}^{\prime}=\left\{\lambda \in \mathbb{R}^{2 m}: \lambda \cdot l \in \mathbb{Z}\right.$ for all $\left.l \in \mathcal{L}\right\}$. Define a linear isomorphism $S: \mathbb{R}^{2 m} \rightarrow \mathbb{R}^{2 m}$ by $S(u) \cdot x=\Omega(u, x)$ for all $x \in \mathbb{R}^{2 m}$. Since $\Omega$ takes integer values on $\mathcal{L} \times \mathcal{L}$, the map $S$ carries $\mathcal{L}$ (injectively) into $\mathcal{L}^{\prime}$. The fact that the invariant factors of $\Omega$ are all equal to one implies that $S(\mathcal{L})=\mathcal{L}^{\prime}$, thus $S$ gives an isomorphism between the lattices $\mathcal{L}$ and $\mathcal{L}^{\prime}$.

The proof of Theorem 1.3 relies on the following result, which will be proven in Appendix C.

4.1. Proposition. Let $L \rightarrow M$ be a line bundle over $M=\mathcal{L} \backslash \mathbb{R}^{2 m}$ with Chern invariant factors $r_{1}=\cdots=$ $r_{m}=1$ and assume that $\mathcal{L}$ has nondegenerate length spectrum. Let $\alpha \in \mathbb{R}^{2 m}$. For $l \in \mathcal{L} \backslash\{0\}$, set

$$
W_{l}^{\alpha}:=e^{-2 \pi i \alpha \cdot l} \int_{\mathcal{F}} d x e^{2 \pi i \Omega(x, l)} \int_{0}^{|l|} d \tau Q\left(x-\tau \frac{l}{|l|}\right)
$$

(Here $\mathcal{F}$ is a fundamental domain for $\mathcal{L} \backslash \mathbb{R}^{2 m}$.) Then $W_{l}^{\alpha}+W_{-l}^{\alpha}$ is an invariant of Spec $(Q ; L)$ for every $l \in \mathcal{L} \backslash\{0\}$. (See Notation 2.35.)

\subsection{COROLlary. Express a potential $Q$ in its Fourier series}

$$
Q(x)=\sum_{\gamma \in \mathcal{L}^{\prime}} c_{\gamma} e^{2 \pi i \gamma \cdot x} .
$$

Under the hypothesis of Proposition 4.1, for each nonzero $\lambda \in \mathcal{L}^{\prime}$, the expression

$$
e^{-2 \pi i \alpha \cdot l} c_{\lambda}+e^{2 \pi i \alpha \cdot l} c_{-\lambda}
$$

where $l=S^{-1}(\lambda)$, is an invariant of $\operatorname{Spec}_{\alpha}(Q ; L)$.

Proof of Corollary 4.2. Let $\lambda \in \mathcal{L}^{\prime}$ and let $l=S^{-1}(\lambda)$, so that $\Omega(l, x)=\lambda \cdot x$ for all $x \in \mathbb{R}^{2 m}$. Then

$$
\begin{aligned}
W_{l}^{\alpha} & =e^{-2 \pi i \alpha \cdot l} \int_{\mathcal{F}} d x e^{-2 \pi i \lambda \cdot x} \int_{0}^{|l|} d \tau\left(\sum_{\gamma \in \mathcal{L}^{\prime}} c_{\gamma} e^{2 \pi i \gamma \cdot x} e^{-2 \pi i \tau \gamma \cdot \frac{l}{|l|}}\right) \\
& =e^{-2 \pi i \alpha \cdot l} \sum_{\gamma \in \mathcal{L}^{\prime}} c_{\gamma} \int_{\mathcal{F}} d x e^{-2 \pi i(\lambda-\gamma) \cdot x} \int_{0}^{|l|} d \tau\left(e^{-2 \pi i \tau \gamma \cdot \frac{l}{|l|}}\right)
\end{aligned}
$$

Since the first integral in each term of the latter sum vanishes when $\gamma \neq \lambda$ and since $\lambda \cdot l=\Omega(l, l)=0$, we thus have $W_{l}^{\alpha}=|l| \operatorname{vol}(\mathcal{F}) e^{-2 \pi i \alpha \cdot l} c_{\lambda}$ and thus

$$
W_{l}^{\alpha}+W_{-l}^{\alpha}=|l| \operatorname{vol}(\mathcal{F})\left(e^{-2 \pi i \alpha \cdot l} c_{\lambda}+e^{2 \pi i \alpha \cdot l} c_{-\lambda}\right) .
$$

The corollary follows. 
4.4. Proposition. [Gi] Let $L$ be a line bundle over the torus $M$, let $\nabla$ be an arbitrary connection on $L$, and let $\Delta$ be the associated Laplacian. Then $\int_{M} Q(x) d x$ and $\int_{M} Q^{2}(x) d x$ are spectral invariants of the Schrödinger operator $\Delta+Q$.

In particular, the spectrum determines the coefficient $c_{0}$ in the Fourier expansion (4.3) of $Q$.

Proof of Proposition 4.4. This result follows immediately from the heat asymptotics computed by P. Gilkey in [Gi]. Indeed, Gilkey considered an arbitrary second order operator $D$ on a vector bundle $V$ over a Riemannian manifold $M$ such that the leading symbol of $D$ is the metric tensor. In his notation, $D=D_{\nabla}-\mathcal{E}$, where $D_{\nabla}$ is the Laplacian associated with the unique connection on $V$ for which $D_{\nabla}-D$ is a zeroth order operator. In our case, $D$ is the Schrödinger operator $\Delta+Q, D_{\nabla}=\Delta$, and $\mathcal{E}=-Q$. Consider the asymptotics of the trace of the heat kernel $e_{Q}$ of the Schrödinger operator $\Delta+Q$ as $t \rightarrow 0^{+}$:

$$
e_{Q}(t, x, x)=\frac{1}{(4 \pi t)^{n / 2}}\left(1+t u_{1}(x)+t^{2} u_{2}(x)+o\left(t^{2}\right)\right) .
$$

The integral $\int_{M} u_{i}(x) d x$ is a spectral invariant of $\Delta+Q$ for each $i$. By Theorem 4.3 in [Gi] and the fact that $M$ is flat, we have $u_{1}=\mathcal{E}=-Q$; hence $\int_{M} Q(x) d x$ is a spectral invariant of $\Delta+Q$. Next $u_{2}=\frac{1}{2} Q^{2}-\frac{1}{6} \Delta Q$ plus terms depending only on the curvature of the connection. Thus by the Divergence Theorem, the $L^{2}$ norm of $Q$ is a spectral invariant.

Proof of Theorem 1.3. We will prove statements (a) and (b) of the theorem for the spectrum of the Schrödinger operator associated with any translation-invariant and $\mathbb{Z}_{2}$-invariant connection. By Propositiion 2.27, such connections are specified by linear functionals $\alpha$ with $\alpha(\mathcal{L}) \subset \frac{1}{2} \mathbb{Z}$.

(a) Assume $\alpha(\mathcal{L}) \subset \frac{1}{2} \mathbb{Z}$. Then for $l \in \mathcal{L}$, we have $e^{2 \pi i \alpha \cdot l}=e^{-2 \pi i \alpha \cdot l}$, and thus by Corollary $4.2, c_{\gamma}+c_{-\gamma}$ is a spectral invariant of $\Delta_{\alpha}+Q$ for all $0 \neq \gamma \in \mathcal{L}^{\prime}$. So is $c_{0}$ by Proposition 4.4. Thus the even part $Q^{+}$of $Q$ is spectrally determined by $\Delta_{\alpha}+Q$.

(b) By (a) and Proposition 4.4, the spectrum of $\Delta_{\alpha}+Q$ determines the $L^{2}$ norms of both $Q$ and of the even part of $Q$, and hence it determines whether $Q$ is even. Thus (b) follows from (a).

4.5. REMARK. Theorem 1.3 together with Proposition 4.4 show that, under the hypotheses of Theorem 1.3, the $L^{2}$ norm of the odd part $Q^{-}=\frac{1}{2}(Q-\check{Q})$ of $Q$ is a spectral invariant of the Schrödinger operator $\Delta+Q$.

Proof of Theorem 1.4. Let $0 \neq l \in \mathcal{L}$. The hypothesis that $(\alpha-\beta) \cdot l$ is irrational implies that the matrix

$$
\left[\begin{array}{ll}
e^{-2 \pi i \alpha \cdot l} & e^{2 \pi i \alpha \cdot l} \\
e^{-2 \pi i \beta \cdot l} & e^{2 \pi i \beta \cdot l}
\end{array}\right]
$$

is invertible. Thus by Corollary 4.2 and Proposition 4.4, from the spectra of $\Delta_{\alpha}+Q$ and $\Delta_{\beta}+Q$ together, we can determine all the Fourier coefficients of $Q$.

4.6. REMARK. Theorem 1.4 contrasts with spectral results for the Schrödinger operator on trivial line bundles. (See Remark 2.38 for notation.) In the case of a trivial bundle, we have the following: (i) Given any smooth real potential $Q$, then $Q$ and $\check{Q}$ necessarily have the same classical Bloch spectrum. (Indeed, given a smooth function $f: \mathbb{R}^{n} \rightarrow \mathbb{C}$ satisfying $(\Delta+Q) f=\mu f$ and the Bloch condition $f(x+l)=e^{-2 \pi i \alpha \cdot l} f(x)$ for all $l \in \mathcal{L}$, let $g:=\breve{f}$ be the complex conjugate of $\check{f}$. Then $g$ satisfies $(\Delta+\check{Q}) g=\mu g$ and $g(x+l)=e^{-2 \pi i \alpha \cdot l} g(x)$ for all $l \in \mathcal{L}$.) Moreover, (ii) Eskin, Ralston and Trubowitz [E-R-T] , Theorem 6.2, proved that if $Q$ is a real analytic potential on a torus with nondegenerate length spectrum, then the spectrum of the Schrödinger operator $\Delta+Q$ acting on functions determines the classical Bloch spectrum of $Q$.

Theorem 1.4 shows that (i) always fails in the setting of line bundles $L$ with Chern invariant factors $r_{1}=\cdots=$ $r_{m}=1$ over tori with nondegenerate length spectrum. Also in this setting, one easily sees that $Q$ and $\check{Q}$ are isospectral with respect to the distinguished connection (and in fact, with respect to any $\mathbb{Z}_{2}$-invariant connection). Thus (ii) also fails. However, we do have that $\operatorname{Spec}_{\alpha}(Q)=\operatorname{Spec}_{-\alpha}(\check{Q})$ for all $\alpha$; indeed the map $g \rightarrow \check{g}$ of 
$C^{\infty}\left(\mathbb{R}^{n}\right)$ intertwines the Schrödinger operators $\Delta_{\varphi^{D}+\alpha}+Q$ and $\Delta_{\varphi^{D}-\alpha}+\check{Q}$. Thus the result (ii) above of Eskin, Ralston, and Trubowitz suggests the following question: Under the hypotheses of Theorems 1.3 and 1.4 , let $P$ and $Q$ be real analytic potentials on $M$ such that $\operatorname{Spec}(P ; L)=\operatorname{Spec}(Q ; L)$. Must it be the case that for each $\alpha \in \mathbb{R}^{n}$, either $\operatorname{Spec}_{\alpha}(P ; L)=\operatorname{Spec}_{\alpha}(Q ; L)$ or $\operatorname{Spec}_{\alpha}(P ; L)=\operatorname{Spec}_{-\alpha}(Q ; L)$ ? If the answer is yes, then Theorem 1.4 would imply for real analytic potentials $Q$ that $I s o(Q ; L)=\{Q, \check{Q}\}$ without any assumptions on the parity of $Q$.

It seems likely that $I s o(Q ; L)=\{Q, \check{Q}\}$ for generic smooth potentials $Q$. This remains an open problem.

Finally, we give an extension of Theorem 1.3 in the case of a 2-dimensional torus:

4.7. Notation. Denote by $\mathcal{S}$ the set of maximal elements in the dual lattice $\mathcal{L}^{\prime}$ of $\mathcal{L}$, i.e. the set of elements $\lambda$ in $\mathcal{L}^{\prime}$ such that $\{\lambda \cdot l \mid l \in \mathcal{L}\}=\mathbb{Z}$. Let $\mathcal{S}_{+} \subset \mathcal{S}$ be a subset with the property that for any $\lambda \in \mathcal{S}, \lambda$ or $-\lambda$ is an element of $\mathcal{S}_{+}$but not both. Then the Fourier decomposition of $Q$

$$
Q(x)=\sum_{\gamma \in \mathcal{L}^{\prime}, \gamma \neq 0} c_{\gamma} e^{2 i \pi \gamma \cdot x}+c_{0}
$$

can be written as (see [E-R-T])

$$
Q(x)=\sum_{\lambda \in \mathcal{S}_{+}} Q_{\lambda}(\lambda \cdot x)+c_{0}
$$

where $Q_{\lambda}$ is the one dimensional potential

$$
Q_{\lambda}(s):=\sum_{k \in \mathbb{Z} \backslash\{0\}} c_{k \lambda} e^{2 \pi i k s} .
$$

Note that if $Q^{ \pm}$are the even and odd parts of the potential $Q$, then $\left(Q^{ \pm}\right)_{\lambda}=\left(Q_{\lambda}\right)^{ \pm}$for all $\lambda \in \mathcal{L}^{\prime}$

4.11. LemmA. Assume the torus $M$ is two-dimensional. Let $\lambda \in \mathcal{S}_{+}$and let $l=S^{-1}(\lambda)$, so that $\Omega(l, x)=\lambda \cdot x$ for all $x$. Let $Q$ be any smooth potential of mean zero (i.e., the Fourier coefficient $c_{0}$ of $Q$ vanishes). Then for $0 \neq k \in \mathbb{Z}$, we have

$$
\int_{0}^{|k l|} d \tau Q\left(x-\tau \frac{l}{|l|}\right)=|k l| Q_{\lambda}(\lambda \cdot x) .
$$

Proof of Lemma 4.11. We have $\lambda \cdot l=\Omega(l, l)=0$ since $\Omega$ is skew-symmetric. Since $M$ is two-dimensional, it follows that for $0 \neq \gamma \in \mathcal{L}^{\prime}$, we have $\gamma \cdot l=0$ only when $\gamma$ is a multiple of $\lambda$. Thus, with $c_{\gamma}, \gamma \in \mathcal{L}^{\prime}$, denoting the Fourier coefficients of $Q$, we have

$$
\int_{0}^{|k l|} d \tau Q\left(x-\tau \frac{l}{|l|}\right)=\sum_{\gamma \in \mathcal{L}^{\prime}} e^{2 \pi i \gamma \cdot x} \int_{0}^{|k l|} d \tau c_{\gamma} e^{-2 \pi i \tau \gamma \cdot \frac{l}{|l|}}=\sum_{0 \neq j \in \mathbb{Z}}|k l| e^{2 \pi i j \lambda \cdot x} c_{j \lambda}=|k l| Q_{\lambda}(\lambda \cdot x) .
$$

4.12. THEOREM. Assume that $M$ is a 2-dimensional torus with nondegenerate length spectrum and $L \rightarrow M$ is a line bundle with Chern invariant factor $r_{1}=1$. Then the squares of the odd parts of the 1-dimensional potentials $Q_{\lambda}$ defined in Notation 4.7 are spectral invariants of $\Delta+Q$; i.e., for any $P \in \operatorname{Spec}(Q ; L)$ and any $\lambda \in \mathcal{S}_{+}$, $\left(P_{\lambda}^{-}\right)^{2}=\left(Q_{\lambda}^{-}\right)^{2}$.

4.13. Corollary. Assume that $M$ is a 2-dimensional torus with nondegenerate length spectrum and $L \rightarrow M$ is a line bundle with Chern invariant factor $r_{1}=1$. Further, assume:

(i) the odd part $Q^{-}$of $Q$ is a 1-dimensional potential, i.e., there exists $\lambda \in \mathcal{S}_{+}$such that $Q^{-}(x)=Q_{\lambda}^{-}(\lambda \cdot x)$, and (ii) $Q_{\lambda}^{-}$is real analytic.

Then $I s o(Q ; L)=\{Q, \check{Q}\}$.

In contrast, for the rectangular two-dimensional tori considered in Theorem 1.1, the examples of $L$-isospectral, noncongruent potentials include real analytic odd one-dimensional potentials. 
Proof of Theorem 4.12. Fix $\lambda \in \mathcal{S}_{+}$and let $l=S^{-1}(\lambda)$. By Theorem C.16 in Appendix C, for every $j \in \mathbb{Z} \backslash\{0\}$, the expression $C_{2, j l}$, given as in Theorem C.19, is a spectral invariant. (Here we are letting the element $j l \in \mathcal{L}$ play the role of $l$ in Theorems C.16 and C.19.) By Lemma C.29 and the assumption that the connection is the distinguished one, the function $a_{0}$ appearing in the expression in Theorem C.19 is given by $a_{0}\left(|j l|, x+j l,-\frac{l}{|l|}\right)=$ $\epsilon_{j l} e^{2 \pi i e_{x}(j l)}$, where $\epsilon_{j l}=e^{-\pi i e_{j l}(j l)}= \pm 1$. Since $\Omega(x, y)=e_{x}(y)-e_{y}(x)$, we have for any $l \in \mathcal{L} \backslash\{0\}$ that $C_{2, j l}$ is a constant multiple of

$$
\int_{\mathcal{F}} d x e^{2 \pi i \Omega(x, j l)}\left(\int_{0}^{|j l|} d \tau Q^{-}\left(x-\tau \frac{l}{|l|}\right)\right)^{2} .
$$

Since $l=S^{-1}(\lambda)$, we have $\Omega(x, j l)=-j \lambda \cdot x$. Thus by Equation (4.14), Lemma 4.11 and the fact that odd potentials always have mean zero, the following expression is a spectral invariant for each $j$ :

$$
\int_{\mathcal{F}} d x e^{-2 \pi i j \lambda \cdot x}|j l|^{2}\left(Q_{\lambda}^{-}(\lambda \cdot x)\right)^{2}
$$

Writing

$$
\left(Q_{\lambda}^{-}(\lambda \cdot x)\right)^{2}=\sum_{0 \neq j \in \mathbb{Z}} b_{j \lambda} e^{2 \pi i j \lambda \cdot x},
$$

the spectral invariant (4.15) simplifies to $\operatorname{vol}(\mathcal{F})|j l|^{2} b_{j \lambda}$. Thus the $b_{j \lambda}$, for $0 \neq j \in \mathbb{Z}$, are spectral invariants, and hence so is the square of the one-dimensional potential $Q_{\lambda}^{-}$. This completes the proof of Theorem 4.12.

\section{Appendices.}

Throughout these appendices, we use the notation of Subsection 2B. In particular, $L$ is a fixed Hermitian line bundle over the torus $M, \nabla$ is a connection on $L$ compatible with the Hermitian structure, $\varphi$ is the 1-form on $\mathbb{R}^{n}$ associated with $\nabla$ as in Definition 2.14, and $\Delta_{\varphi}$ is the associated Laplacian, given as in Proposition 2.33.

While we are interested primarily in translation-invariant connections, we make no assumption on the connection in Appendices A-C, except where specified at the end of Appendix C.

\section{APPENDIX A. WAVE KERNEL ON $\mathbb{R}^{n}$.}

Consider the wave equation on $\mathbb{R}^{n}, n \geq 2$, with smooth potential $Q: \mathbb{R}^{n} \rightarrow \mathbb{R}$

$$
\left(\square_{\varphi}+Q(x)\right) u(t, x)=0 \quad\left(x \in \mathbb{R}^{n}, t \in \mathbb{R}\right)
$$

with initial conditions

$$
u(0, x)=u_{0}(x) ; \quad \partial_{t} u(0, x)=0,
$$

where $u_{0} \in C^{\infty}\left(\mathbb{R} \times \mathbb{R}^{n}, \mathbb{C}\right)$ and

$$
\square_{\varphi}=\frac{\partial^{2}}{\partial t^{2}}+\Delta_{\varphi}
$$

Denote by $K(t, x, y)$ the distributional wave kernel and $K(t)$ the wave operator corresponding to (A.1) and (A.2); i.e., for any choice of $u_{0}$, the function

$$
u(t, x)=\left(K(t) u_{0}\right)(x):=\int_{\mathbb{R}^{n}} K(t, x, y) u_{0}(y) d y
$$

solves (A.1) - (A.2). The operator $K(t)$ can be written as the sum of two integral operators $\frac{1}{2}\left(K_{+}(t)+K_{-}(t)\right)$ where $K_{ \pm}$has a symbol of the form $\frac{1}{(2 \pi)^{n}} e^{i S^{ \pm}} a^{ \pm}$with $S^{ \pm}=S^{ \pm}(t, x, y, \zeta)$ denoting the phase function of $K_{ \pm}$ and $a^{ \pm}=a^{ \pm}(t, x, \zeta)$ its amplitude. (Here $t \in \mathbb{R}$ and $x, y, \zeta \in \mathbb{R}^{n}$.) As $S^{ \pm}(t, x, y, \zeta)$ only depends on the principal 
symbol of $\Delta_{\varphi}+Q$, which is independent of $\varphi$ and $Q, S^{ \pm}$are the corresponding phase functions for the free wave equation on $\mathbb{R}^{n}$, i.e.

$$
S^{ \pm}(t, x, y, \zeta)=(x-y) \cdot \zeta \pm t|\zeta|
$$

Thus

$$
\left(K_{ \pm}(t) u_{0}\right)(x)=\frac{1}{(2 \pi)^{n}} \int_{\mathbb{R}^{n}} d \zeta \int_{\mathbb{R}^{n}} d y e^{i(x-y) \cdot \zeta \pm i t|\zeta|} a^{ \pm}(t, x, \zeta) u_{0}(y) .
$$

The amplitudes $a^{ \pm}$satisfy the following estimates: for any choice of multi-indices $\alpha=\left(\alpha_{j}\right)_{1 \leq j \leq n}, \beta=$ $\left(\beta_{j}\right)_{1 \leq j \leq n}$ and any choice of $R>0$ and $T>0$, there exists a constant $C_{\alpha, \beta}=C_{\alpha, \beta}(R, T)$ such that for any $\zeta \in \mathbb{R}^{n}$,

$$
\sup _{0 \leq t \leq T,|x|<R}\left|\partial_{x}^{\alpha} \partial_{\zeta}^{\beta} a^{ \pm}(t, x, \zeta)\right| \leq C_{\alpha, \beta}(1+|\zeta|)^{-|\beta|}
$$

where $|\beta|=\beta_{1}+\cdots+\beta_{n}$. Moreover they satisfy the initial conditions $a^{ \pm}(0, x, \zeta)=1$.

Following [La] (see [E-R-T] for the case $\varphi \equiv 0$ ), we approximate $K_{ \pm}(t)$ by

$$
\left(K_{ \pm}^{N}(t) u_{0}\right)(x)=\frac{1}{(2 \pi)^{n}} \int_{\mathbb{R}^{n}} d \zeta \int_{\mathbb{R}^{n}} d y e^{i(x-y) \cdot \zeta \pm i t|\zeta|}\left(a_{0}^{ \pm}+\cdots+a_{N}^{ \pm}\right)(t, x, \zeta) \chi(|\zeta|) u_{0}(y)
$$

where $a_{j}^{ \pm}=a_{j}^{ \pm}(t, x, \zeta)$ is positive-homogeneous of degree $-j$ in $\zeta$, i.e. $\forall c>0, t>0, x \in \mathbb{R}^{n}, \zeta \in \mathbb{R}^{n}, a_{j}^{ \pm}(t, x, c \zeta)=$ $c^{-j} a_{j}^{ \pm}(t, x, \zeta)$, and $a_{j}^{ \pm}$satisfies the initial conditions

$$
a_{0}^{ \pm}(0, x, \zeta)=1 \text { and } a_{j}^{ \pm}(0, x, \zeta)=0(1 \leq j \leq N) .
$$

Here $\chi(r)$ is a smooth cut-off function of the form

$$
\chi(r)=\left\{\begin{array}{llr}
0 & \text { for } & -\infty<r \leq 1 / 2 \\
1 & \text { for } & 1 \leq r<\infty
\end{array}\right.
$$

The functions $a_{j}^{ \pm}(t, x, \zeta)$ are determined inductively by solving transport equations. For any given $M \geq 0$ we will choose the integer $N \geq 0$ so large that the integral operators $\widetilde{K}_{ \pm}(t)$ defined by

$$
\left(\square_{\varphi}+Q\right) K_{ \pm}^{N}(t) u_{0}=\widetilde{K}_{ \pm}(t) u_{0}
$$

have $C^{M}$ kernels. Indeed, $\left(\square_{\varphi}+Q\right) K_{ \pm}^{N}(t) u_{0}(x)$ equals

$$
\frac{1}{(2 \pi)^{n}} \int_{\mathbb{R}^{n}} d \zeta \int_{\mathbb{R}^{n}} d y \quad\left(\square_{\varphi}+Q(x)\right)\left(e^{i(x-y) \cdot \zeta \pm i t|\zeta|}\left(a_{0}^{ \pm}+\cdots+a_{N}^{ \pm}\right)(t, x, \zeta)\right) \chi(|\zeta|) u_{0}(y)
$$

where $\square_{\varphi}$ is computed with respect to the variables $t$ and $x$. By Proposition 2.33, using the notation $\left.\varphi\right|_{x}$ established in Remark 2.17,

$$
\square_{\varphi} \cdot e^{i(x-y) \cdot \zeta \pm i t|\zeta|}=\left(4 \pi \varphi_{\mid x}(\zeta)+4 \pi^{2}\left|\varphi_{\mid x}\right|^{2}-2 \pi i \operatorname{div}\left(\varphi^{\#}\right)(x)\right) e^{i(x-y) \cdot \zeta \pm i t|\zeta|},
$$

where $\left.\phi\right|_{x}$ is viewed as a linear functional on $\mathbb{R}^{n}$ as in Remark 2.17. Further one has the product formula

$$
\square_{\varphi}(A B)=\left(\square_{\varphi} A\right) B+A\left(\square_{\varphi} B\right)-4 \pi^{2}|\varphi|^{2} A B+2 \frac{\partial A}{\partial t} \frac{\partial B}{\partial t}-2 \operatorname{grad} A \cdot \operatorname{grad} B+2 \pi i A B \operatorname{div}\left(\varphi^{\#}\right)
$$

Hence

$$
\begin{gathered}
\left(\square_{\varphi}+Q(x)\right)\left(e^{i(x-y) \cdot \zeta \pm i t|\zeta|}\left(a_{0}^{ \pm}+\cdots+a_{N}^{ \pm}\right)(t, x, \zeta)\right) \\
=e^{i(x-y) \cdot \zeta \pm i t|\zeta|}\left(\left.4 \pi \varphi\right|_{x}(\zeta) \pm 2 i|\zeta| \frac{\partial}{\partial t}-2 i \zeta \cdot \operatorname{grad}+\left(\square_{\varphi}+Q(x)\right)\right) \cdot\left(a_{0}^{ \pm}+\cdots+a_{N}^{ \pm}\right)(t, x, \zeta) \\
=e^{i(x-y) \cdot \zeta \pm i t|\zeta|}\left[\left(\left.4 \pi \varphi\right|_{x}(\zeta) a_{0}^{ \pm}(t, x, \zeta) \pm 2 i|\zeta| \frac{\partial a_{0}^{ \pm}}{\partial t}(t, x, \zeta)-2 i \zeta \cdot \operatorname{grad} a_{0}^{ \pm}(t, x, \zeta)\right)\right. \\
+\sum_{j=1}^{N}\left(\left.4 \pi \varphi\right|_{x}(\zeta) a_{j}^{ \pm}(t, x, \zeta) \pm 2 i|\zeta| \frac{\partial a_{j}^{ \pm}}{\partial t}(t, x, \zeta)-2 i \zeta \cdot \operatorname{grad} a_{j}^{ \pm}(t, x, \zeta)+\left(\square_{\varphi}+Q(x)\right) a_{j-1}^{ \pm}(t, x, \zeta)\right)
\end{gathered}
$$




$$
\left.+\left(\square_{\varphi}+Q(x)\right) a_{N}^{ \pm}(t, x, \zeta)\right]
$$

where $\operatorname{grad} a_{j}^{ \pm}(t, x, \zeta)$ refers to the gradient with respect to $x$. Thus we are led to the transport equations

$$
\left\{\begin{array}{l}
\left.4 \pi \varphi\right|_{x}(\zeta) a_{0}^{ \pm}(t, x, \zeta) \pm 2 i|\zeta| \frac{\partial a_{0}^{ \pm}}{\partial t}(t, x, \zeta)-2 i \zeta \cdot \operatorname{grad} a_{0}^{ \pm}(t, x, \zeta)=0 \\
a_{0}^{ \pm}(0, x, \zeta)=1
\end{array}\right.
$$

and for $1 \leq j \leq N$,

$$
\left\{\begin{array}{l}
\left.4 \pi \varphi\right|_{x}(\zeta) a_{j}^{ \pm}(t, x, \zeta) \pm 2 i|\zeta| \frac{\partial a_{j}^{ \pm}}{\partial t}(t, x, \zeta)-2 i \zeta \cdot \operatorname{grad} a_{j}^{ \pm}(t, x, \zeta)=-\left(\square_{\varphi}+Q(x)\right) a_{j-1}^{ \pm}(t, x, \zeta) \\
a_{j}^{ \pm}(0, x, \zeta)=0
\end{array}\right.
$$

Note that the solution $a_{0}^{+}(t, x, \zeta)$ of (A.9) with + has the property that $a_{0}^{+}(-t, x, \zeta)$ satisfies (A.9) with -. By uniqueness, it then follows that $a_{0}^{-}(t, x, \zeta)=a_{0}^{+}(-t, x, \zeta)$. As a consequence, $\square_{\varphi} a_{0}^{+}(-t, x, \zeta)=\square_{\varphi} a_{0}^{-}(t, x, \zeta)$ and hence by the same argument, Equation (A.10) implies that $a_{1}^{-}(t, x, \zeta)=a_{1}^{+}(-t, x, \zeta)$ and, by induction, that $a_{j}^{-}(t, x, \zeta)=a_{j}^{+}(-t, x, \zeta)$ for any $j \geq 0$. Hence, for any $j \geq 0$,

$$
\left.\frac{\partial}{\partial t}\right|_{t=0}\left(a_{j}^{+}(t, x, \zeta)+a_{j}^{-}(t, x, \zeta)\right)=0
$$

Equation (A.7) is then satisfied with the kernel of the integral operator $\widetilde{K}_{ \pm}(t)$ given by

$$
\widetilde{K}_{ \pm}(t, x, y):=\frac{1}{(2 \pi)^{n}} \int_{\mathbb{R}^{n}} d \zeta e^{i(x-y) \cdot \zeta \pm i t|\zeta|}\left(\left(\square_{\varphi}+Q(x)\right) a_{N}^{ \pm}(t, x, \zeta)\right) \chi(|\zeta|) .
$$

As $a_{N}^{ \pm}$is positive-homogeneous of degree $-N$ in $\zeta$, so is $\left(\square_{\varphi}+Q\right) a_{N}^{ \pm}$. Hence, for each of $\widetilde{K}_{ \pm}$, it follows that the integral in (A.12) is convergent for $N \geq n+1$ and, given any $M$, there exists $N \geq n+1$ so that it is a $C^{M}$ function of $x$ and $y$, as required.

We are now able to check the accuracy of the approximation of $K$ by $K^{N}:=\frac{1}{2}\left(K_{+}^{N}+K_{-}^{N}\right)$. Since

$$
\left.\frac{d}{d t}\right|_{t=0}\left[K^{N}(t) u_{0}\right](x)=\frac{1}{2} \frac{1}{(2 \pi)^{n}} \int_{\mathbb{R}^{n}} d \zeta \int_{\mathbb{R}^{n}} d y e^{i(x-y) \cdot \zeta} \sum_{j=0}^{N}\left(\frac{\partial}{\partial t} a_{j}^{+}+\frac{\partial}{\partial t} a_{j}^{-}\right)(0, x, \zeta) \chi(|\zeta|) u_{0}(y),
$$

Equation (A.11) implies that

$$
\left.\frac{\partial}{\partial t}\right|_{t=0} K^{N}(t) u_{0}=0
$$

Moreover, by (A.5)

$$
K^{N}(0) u_{0}(x)=\frac{1}{(2 \pi)^{n}} \int_{\mathbb{R}^{n}} d \zeta \int_{\mathbb{R}^{n}} d y e^{i(x-y) \cdot \zeta} \chi(|\zeta|) u_{0}(y) .
$$

Hence, with $\widehat{u_{0}}$ denoting the Fourier transform of $u_{0}$,

$$
\begin{aligned}
K^{N}(0) u_{0}(x) & =\frac{1}{(2 \pi)^{n}} \int_{\mathbb{R}^{n}} d \zeta e^{i x \cdot \zeta} \widehat{u_{0}}(\zeta)+\frac{1}{(2 \pi)^{n}} \int_{\mathbb{R}^{n}} d \zeta \int_{\mathbb{R}^{n}} d y e^{i(x-y) \cdot \zeta}(\chi(|\zeta|)-1) u_{0}(y) \\
& =u_{0}(x)+\frac{1}{(2 \pi)^{n}} \int_{\mathbb{R}^{n}} d \zeta \int_{\mathbb{R}^{n}} d y e^{i(x-y) \cdot \zeta}(\chi(|\zeta|)-1) u_{0}(y),
\end{aligned}
$$

by the inverse Fourier transform theorem in $L^{2}\left(\mathbb{R}^{n}\right)$. Thus

$$
K^{N}(0) u_{0}=u_{0}+K_{0}^{N} u_{0}
$$

where $K_{0}^{N}$ is an integral operator with a smooth kernel.

Denoting by $\underline{K}^{N}$ the difference $K^{N}-K$, we are led to the following Cauchy problem

$$
\left\{\begin{array}{l}
\left(\square_{\varphi}+Q\right) \underline{K}^{N}(t) u_{0}=\widetilde{K}(t) u_{0} \\
\underline{K^{N}}(0) u_{0}=K_{0}^{N} u_{0} \\
\left.\frac{\partial}{\partial t}\right|_{t=0} \underline{K}^{N}(t) u_{0}=0
\end{array}\right.
$$


where $\widetilde{K}(t)=\frac{1}{2}\left(\widetilde{K}_{+}+\widetilde{K}_{-}\right)$has a $C^{M}$ kernel for a given $M$, provided $N$ is sufficiently large and where $K_{0}^{N}$ has a smooth kernel. By regularity theory for solutions of (A.15), $\underline{K}^{N}(t)$ is an integral operator with a $C^{M^{\prime}}$ kernel, for any given $M^{\prime}$, provided $N$ is large enough. This will be useful in Appendix C (see the proof of Proposition C.11).

We now solve (A.9) and (A.10). As we will only need the $a_{j}^{+}$'s we compute only these coefficients. Let

$$
a_{j}=\left.a_{j}^{+}\right|_{\mathbb{R}^{+} \times \mathbb{R}^{n} \times S^{n-1}} .
$$

Since $a_{j}^{+}(t, x, \zeta)$ is positive-homogeneous in $\zeta$, it suffices to solve for the functions $a_{j}$. Dividing (A.9) by $2 i$ and restricting to $\mathbb{R}^{+} \times \mathbb{R}^{n} \times S^{n-1}$, one gets

$$
\left\{\begin{array}{l}
\frac{\partial a_{0}}{\partial t}(t, x, \omega)-\omega \cdot \operatorname{grad} a_{0}(t, x, \omega)-\left.2 \pi i \varphi\right|_{x}(\omega) a_{0}(t, x, \omega)=0 \\
a_{0}(0, x, \omega)=1
\end{array}\right.
$$

\section{A.18. LEMMA. Equation (A.17) has the following solution}

$$
a_{0}(t, x, \omega)=\exp \left(\left.2 \pi i \int_{0}^{t} \varphi\right|_{(x+\tau \omega)}(\omega) d \tau\right) .
$$

The function $a_{0}$ is smooth in all its arguments.

Proof. First note that if $h \in C^{\infty}\left(\mathbb{R}^{n}\right)$, then for fixed $\omega$, the function $g(t, x)=\exp \left(2 \pi i \int_{0}^{t} h(x+\tau \omega) d \tau\right)$ satisfies (with grad denoting the gradient with respect to $x$ ):

$$
\begin{gathered}
\omega \cdot \operatorname{grad} g(t, x)=g(t, x) 2 \pi i \int_{0}^{t} \omega \cdot \operatorname{grad}(h)(x+\tau \omega) d \tau \\
=g(t, x) 2 \pi i \int_{0}^{t} \frac{\partial}{\partial \tau} h(x+\tau \omega) d \tau \\
=g(t, x) 2 \pi i h(x+t \omega)-g(t, x) 2 \pi i h(x) \\
=\frac{\partial g}{\partial t}(t, x)-2 \pi i h(x) g(t, x) .
\end{gathered}
$$

Moreover $g(0, x)=1$. Let $h(t, x)=\left.\varphi\right|_{(x+\tau \omega)}(\omega)$ (again viewing $\omega$ as arbitrary but fixed) to see that the function defined in Equation (A.19) satisfies (A.17).

Similarly, dividing (A.10) by $2 i$, we obtain in view of (A.16)

$$
\left\{\begin{array}{l}
\frac{\partial a_{j}}{\partial t}(t, x, \omega)-\omega \cdot \operatorname{grad} a_{j}(t, x, \omega)-\left.2 \pi i \varphi\right|_{x}(\omega) a_{j}(t, x, \omega)=\frac{i}{2}\left(\square_{\varphi}+Q(x)\right) a_{j-1}(t, x, \omega) \\
a_{j}(0, x, \omega)=0
\end{array}\right.
$$

We solve (A.20) by the method of variation of constants. First for $0 \leq j \leq N$, define $f_{j}: \mathbb{R}^{+} \times \mathbb{R}^{n} \times S^{n-1} \rightarrow \mathbb{R}$ by

$$
f_{j}(t, x, \omega)=\frac{a_{j}(t, x, \omega)}{a_{0}(t, x, \omega)} .
$$

By (A.17) and (A.21), we may rewrite the system (A.20) for $1 \leq j \leq N$ as

$$
\left\{\begin{array}{l}
\frac{\partial f_{j}}{\partial t}-\omega \cdot \operatorname{grad} f_{j}=\frac{i}{2 a_{0}}\left(\square_{\varphi}+Q\right) a_{0} f_{j-1} \\
f_{j}(0, x, \omega)=0 .
\end{array}\right.
$$

To solve (A.22) we notice that

Hence

$$
\frac{d}{d t} f_{j}(t, x-\omega t, \omega)=\left(\frac{\partial f_{j}}{\partial t}-\omega \cdot \operatorname{grad} f_{j}\right)(t, x-\omega t, \omega) .
$$

$$
f_{j}(t, x-\omega t, \omega)=\frac{i}{2} \int_{0}^{t}\left(\frac{\square_{\varphi}\left(a_{0} f_{j-1}\right)}{a_{0}}+Q f_{j-1}\right)(\tau, x-\tau \omega, \omega) d \tau
$$


or

$$
f_{j}(t, x, \omega)=\frac{i}{2} \int_{0}^{t}\left(\frac{\square_{\varphi}\left(a_{0} f_{j-1}\right)}{a_{0}}+Q f_{j-1}\right)(\tau, x+(t-\tau) \omega, \omega) d \tau .
$$

Inductively, one proves that $f_{j}$ is smooth in all its arguments.

As $f_{0} \equiv 1$, formula (A.23) leads for $j=1$ to the decomposition

$$
f_{1}=f_{1,0}+f_{1,1}
$$

of $f_{1}$, where

$$
f_{1,0}(t, x, \omega):=\frac{i}{2} \int_{0}^{t} \frac{\square_{\varphi} a_{0}}{a_{0}}(\tau, x+(t-\tau) \omega, \omega) d \tau
$$

and

$$
f_{1,1}(t, x, \omega):=\frac{i}{2} \int_{0}^{t} Q(x+(t-\tau) \omega) d \tau \stackrel{\text { change of variable }}{=} \frac{i}{2} \int_{0}^{t} Q(x+\tau \omega) d \tau .
$$

Note that $f_{1,0}$ is independent of $Q$ whereas $f_{1,1}$ is linear in $Q$.

Equations (A.21) and (A.24) yield a decomposition for $a_{1}$ :

$$
a_{1}=a_{1,0}+a_{1,1}
$$

where for $j=1,0$,

$$
a_{1, j}:=a_{0} f_{1, j}
$$

Similarly, one has

$$
f_{2}=f_{2,0}+f_{2,1}+f_{2,2}
$$

and

$$
a_{2}=a_{2,0}+a_{2,1}+a_{2,2}
$$

where $a_{2, j}:=a_{0} f_{2, j}(\forall j=0,1,2)$ and

$$
\begin{gathered}
f_{2,0}(t, x, \omega):=\frac{i}{2} \int_{0}^{t}\left(\frac{\square_{\varphi}\left(a_{0} f_{1,0}\right)}{a_{0}}\right)(\tau, x+(t-\tau) \omega, \omega) d \tau, \\
f_{2,1}(t, x, \omega)=\frac{i}{2} \int_{0}^{t}\left(\frac{\square_{\varphi}\left(a_{0} f_{1,1}\right)}{a_{0}}+Q f_{1,0}\right)(\tau, x+(t-\tau) \omega, \omega) d \tau
\end{gathered}
$$

and

$$
f_{2,2}(t, x, \omega)=\frac{i}{2} \int_{0}^{t} Q f_{1,1}(\tau, x+(t-\tau) \omega, \omega) d \tau
$$

Substituting (A.26) into (A.33) and changing variables, we obtain

$$
\begin{aligned}
f_{2,2}(t, x, \omega) & =-\frac{1}{4} \int_{0}^{t}\left[Q(x+(t-\tau) \omega) \int_{0}^{\tau} Q(x+(t-s) \omega) d s\right] d \tau \\
& =-\frac{1}{4} \int_{0}^{t} \frac{1}{2} \frac{d}{d \tau}\left(\int_{0}^{\tau} Q(x+(t-s) \omega) d s\right)^{2} d \tau \\
& =-\frac{1}{8}\left(\int_{0}^{t} Q(x+(t-\tau) \omega) d \tau\right)^{2} \\
& =-\frac{1}{8}\left(\int_{0}^{t} Q(x+\tau \omega) d \tau\right)^{2}
\end{aligned}
$$

Finally we want to analyze the symmetries of the functions $a_{0}$ and $f_{j}$ under the assumptions that the connection is $\mathbb{Z}_{2}$-invariant (see Definition 2.21) and that the $\mathcal{L}$-periodic potential $Q$ is even.

A.35. Proposition. Assume that the connection $\nabla$ is $\mathbb{Z}_{2}$-invariant. Then for any $t>0, x \in \mathbb{R}^{n}$ and $\omega \in S^{n-1}$,

(i) $a_{0}(t,-x,-\omega)=a_{0}(t, x, \omega)$

(ii) If in addition $Q$ is even, one has for any $j \geq 1, f_{j}(t,-x,-\omega)=f_{j}(t, x, \omega)$. 
Proof. Statement (i) follows from formula (A.19) and Definition 2.21. Statement (ii) follows from an induction argument using (A.23), Remark 2.32, and statement (i).

\section{APPENDIX B. WAVE KERNEL ACTING ON SECTIONS OF A LINE BUNDLE.}

In this appendix we show how to construct the wave kernel acting on sections of the complex line bundle $L$ over the $n$-dimensional torus $M=\mathcal{L} \backslash \mathbb{R}^{n}, n \geq 2$, from the wave kernel on $\mathbb{R}^{n}$ constructed in section A.

Recall that sections of $L \rightarrow M$ may be viewed equivalently as functions $f: \mathbb{R}^{n} \rightarrow \mathbb{C}$ satisfying Equation (2.12) for all $l \in \mathcal{L}$, where $e_{l}$ is defined as in Notation 2.4.

We introduce the following distributional kernel

$$
K_{L}(t, x, y):=\sum_{l \in \mathcal{L}} e^{-2 \pi i e_{l}(x)} K(t, x+l, y),
$$

where $K(t, x, y)$ denotes the wave kernel on $\mathbb{R}^{n}$ introduced in the previous section. For any section $u_{0}$ of $L$, again viewed as a function $u_{0}: \mathbb{R}^{n} \rightarrow \mathbb{C}$ satisying Equation (2.12), define

$$
\begin{aligned}
u(t, x) & :=\int_{\mathcal{F}} K_{L}(t, x, y) u_{0}(y) d y \\
& =\sum_{l \in \mathcal{L}} e^{-2 \pi i e_{l}(x)} \int_{\mathcal{F}} K(t, x+l, y) u_{0}(y) d y,
\end{aligned}
$$

where $\mathcal{F}$ denotes a fixed fundamental domain of the action of $\mathcal{L}$ on $\mathbb{R}^{n}$. By a standard domain of dependence argument the sum in (B.2) is finite for any given $x$ and $t$.

We claim that $u(t, x)$ satisfies (A.1) - (A.2) as well as (2.12), i.e., one has the following:

B.3. Proposition. For any $x \in \mathbb{R}^{n}, t>0$,

(i) $\left(\square_{\varphi}+Q\right)(x) \cdot u(t, x)=0$

(ii) $u(0, x)=u_{0}(x) ; u_{t}(0, x)=0$

(iii) $u(t, x+l)=e^{2 \pi i e_{l}(x)} u(t, x)$ for all $l \in \mathcal{L}$.

Proof.

(i) The group $\mathcal{L}$ acts on $C^{\infty}\left(\mathbb{R}^{+} \times \mathbb{R}^{n}, \mathbb{C}\right)$ by $(l . v)(t, x)=e^{2 \pi i e_{l}(x)} v(t, x-l)$. By Remark 2.32, this action commutes with $\Delta_{\varphi}$ and hence with the Schrödinger operator $\left(\square_{\varphi}+Q\right)$. Assertion (i) follows.

(ii) Letting $t$ approach 0 in $\sum_{l \in \mathcal{L}} e^{-2 \pi i e_{l}(x)} \int_{\mathcal{F}} K(t, x+l, y) u_{0}(y) d y$, one gets by (B.2) that for any $x \in \mathbb{R}^{n}$,

$$
u(0, x)=\sum_{l \in \mathcal{L}} e^{-2 \pi i e_{l}(x)} \int_{\mathcal{F}} \delta_{x+l}(y) u_{0}(y) d y=e^{-2 \pi i e_{l_{x}}(x)} u_{0}\left(x+l_{x}\right)
$$

where $\delta$ denotes the Dirac delta function and $l_{x}$ is the unique element in $\mathcal{L}$ such that $x+l_{x} \in \mathcal{F}$. Since $u_{0}\left(x+l_{x}\right)=$ $e^{2 \pi i e_{l_{x}}(x)} u_{0}(x)$, one thus obtains $u(0, x)=u_{0}(x)$. Moreover, as $\left.\frac{\partial}{\partial t}\right|_{t=0} K \equiv 0$, one has

$$
\frac{\partial u}{\partial t}(0, x)=\left.\sum_{l \in \mathcal{L}} e^{-2 \pi i e_{l}(x)} \int_{\mathcal{F}} \frac{\partial}{\partial t}\right|_{t=0} K(t, x+l, y) u_{0}(y) d y=0 .
$$

(iii) This is immediate since $u$ is invariant under the action of $\mathcal{L}$ defined in (i).

\section{APPENDIX C. EXPANSION OF THE WAVE TRACE AT SINGULARITIES.}

In this appendix, we analyze the singularities of the wave trace $W_{L}$. This is a distribution in the variable $t$ which when applied to $f \in C_{0}^{\infty}(\mathbb{R})$ is given by

$$
\left\langle W_{L}, f\right\rangle=\int_{\mathcal{F}} d x \int_{\mathbb{R}} d t f(t) K_{L}(t, x, x)
$$

where $\mathcal{F}$ again denotes a fixed fundamental domain of the action of $\mathcal{L}$ on $\mathbb{R}^{n}$ invariant under the $\mathbb{Z}_{2}$-action. 
Eskin, Ralston and Trubowitz [E-R-T] analyzed the singularities of the wave trace in the case of trivial line bundles over tori (i.e., for the Schrödinger operator acting on smooth functions on the torus). We will use the same approach in our setting. We continue to assume throughout this section that $n \geq 2$.

In view of Proposition B.3, we have

$$
\left\langle W_{L}, f\right\rangle=\int_{\mathcal{F}} d x \int_{\mathbb{R}} d t f(t) \sum_{l \in \mathcal{L}} e^{-2 \pi i e_{l}(x)} K(t, x+l, x),
$$

where by a standard domain of dependence argument, the sum in (C.2) is finite for any $x \in \mathbb{R}^{n}$ and $t>0$. Hence

$$
\left\langle W_{L}, f\right\rangle=\int_{\mathcal{F}} d x \sum_{l \in \mathcal{L}} e^{-2 \pi i e_{l}(x)} \int_{\mathbb{R}} d t f(t) K(t, x+l, x),
$$

where the sum is again finite, as $f$ has compact support.

The singular support of the distribution $W_{L}$ is given by the set $\{|l|: l \in \mathcal{L}\}$. By [D-G], the contributions to the singularity of $W_{L}$ at $t=|d|$ come from

$$
\int_{\mathcal{F}} d x \sum_{l \in \mathcal{L},|l|=|d|} e^{-2 \pi i e_{l}(x)} K(t, x+l, x) .
$$

For $d \in \mathcal{L} \backslash\{0\}$, let $\beta_{|d|}$ be a smooth, compactly supported function on $\mathbb{R}$ such that $\beta_{|d|}(t)=1$ for $t$ near $|d|$, $\operatorname{supp}\left(\beta_{|d|}\right) \subset \mathbb{R}^{+}$, and $\operatorname{supp}\left(\beta_{|d|}\right) \cap|\mathcal{L}|=\{|d|\}$. Then the singular support of the distribution $\beta_{|d|} W_{L}$ is given by $\{ \pm|d|\}$. Hence, by (C.4) and the assumption that $\mathcal{L} \backslash \mathbb{R}^{n}$ has simple length spectrum, the Fourier transform $\widehat{\beta_{|d|} W_{L}}$ of $\beta_{|d|} W_{L}$ with respect to time $t$ satisfies

$$
\widehat{\beta_{|d|} W_{L}}(\eta) \stackrel{\eta \rightarrow+\infty}{=} \sum_{l= \pm d} \int_{\mathcal{F}} d x \int_{\mathbb{R}} d t e^{-i t \eta} \beta_{|d|}(t) e^{-2 \pi i e_{l}(x)} K(t, x+l, x)+O\left(\eta^{-\infty}\right) .
$$

C.6. Definition. For $l \in \mathcal{L} \backslash\{0\}, x \in \mathbb{R}^{n}, \omega \in S^{n-1}$ and $k$ any nonnegative integer, set

$$
b_{k, l, \beta}(x, \omega)=\left.\sum_{j=0}^{k}\left(\begin{array}{c}
n-1-j \\
k-j
\end{array}\right)\left(i \frac{\partial}{\partial t}\right)^{k-j}\right|_{t=\omega \cdot l} \beta_{|l|}(t) a_{j}(t, x+l,-\omega)
$$

and let

$$
b_{k, l}(x, \omega)=\left.\sum_{j=0}^{k}\left(\begin{array}{c}
n-1-j \\
k-j
\end{array}\right)\left(i \frac{\partial}{\partial t}\right)^{k-j}\right|_{t=\omega \cdot l} a_{j}(t, x+l,-\omega) .
$$

(To review the notion of the binomial coefficients $\left(\begin{array}{c}-m \\ r\end{array}\right)$ when $m$ and $r$ are positive, see Equation (D.2) in Appendix D.)

In particular,

$$
b_{0, l}(x, \omega)=a_{0}(\omega \cdot l, x+l,-\omega)
$$

For any integer $R \geq 1$, set

$$
J_{l, R}(\eta):=\frac{1}{(2 \pi)^{n-1}} \sum_{k=0}^{R+n-2} \eta^{n-1-k} \int_{\mathcal{F}} d x e^{-2 \pi i e_{l}(x)} \int_{S_{+}^{n-1}(l)} d_{v o l} \omega e^{-i \eta \omega \cdot l} b_{k, l, \beta}(x, \omega)
$$

where $d_{v o l} \omega$ is the volume form on $S^{n-1}$ and $S_{+}^{n-1}(l):=\left\{\omega \in S^{n-1} \mid \omega \cdot l>0\right\}$.

C.11. Proposition. Assume that $\mathcal{L} \backslash \mathbb{R}^{n}$ has simple length spectrum. Then for any $R \geq 1$,

$$
\widehat{\beta_{|d|} W_{L}}(\eta) \stackrel{\eta \rightarrow+\infty}{=} \frac{1}{2}\left(J_{d, R}(\eta)+J_{-d, R}(\eta)\right)+O\left(\eta^{-R}\right)
$$


The proof of Proposition C.11 can be found in Appendix D.

To get an asymptotic expansion of $J_{l, R}(l= \pm d)$ we use the method of stationary phase. Note that the map

$$
\begin{array}{ll}
S_{+}^{n-1}(l) & \rightarrow \mathbb{R} \\
\omega & \mapsto-\omega \cdot l
\end{array}
$$

has $\omega=\frac{l}{|l|}$ as the unique critical point and that this point is a minimum. Thus the asymptotic expansion as $\eta \rightarrow+\infty$ of the integral $\int_{S_{+}^{n-1}(l)} e^{-i \eta \omega \cdot l} b_{k, l, \beta}(x, \omega) d_{v o l} \omega$ is related to the behavior of $b_{k, l, \beta}(x, \omega)$ with $\omega$ restricted to an arbitrarily small neighborhood of this point. Since $\beta_{|l|}(\omega \cdot l)$ is identically one for $\omega$ in some neighborhood $U$ of $\frac{l}{|l|}$ in $S^{n-1}$, we have $b_{k, l}(x, \omega)=b_{k, l, \beta}(x, \omega)$ when $\omega \in U$. Thus in computing the asymptotic expansion, we may work with the functions $b_{k, l}$ rather than $b_{k, l, \beta}$.

We can give explicit Morse coordinates on the open hemisphere $S_{+}^{n-1}(l)$. First, let $\left(y_{1}, \ldots, y_{n}\right)$ be the standard rectangular coordinates on $\mathbb{R}^{n}$, rotated so that the coordinates of $\frac{l}{|l|}$ are $(0, \ldots, 0,1)$. Define coordinates $z=$ $\left(z_{1}, \ldots, z_{n-1}\right)$ on $S_{+}^{n-1}(l)$ by setting

$$
\left(z_{1}, \ldots, z_{n-1}\right)=\sqrt{\frac{2}{1+y_{n}}}\left(y_{1}, \ldots, y_{n-1}\right) .
$$

These coordinates map $S_{+}^{n-1}(l)$ onto a ball of radius $\sqrt{2}$ about the origin in $\mathbb{R}^{n-1}$ and carry $\frac{l}{|l|}$ to 0 . We will denote by $\omega(z)$ the point in $S_{+}^{n-1}(l)$ with coordinates $z$.

C.12. Lemma. Let $z=\left(z_{1}, \ldots, z_{n-1}\right)$ be the coordinate chart on $S_{+}^{n-1}(l)$ defined above. Then:

(i) $\omega(z) \cdot l=|l|\left(1-|z|^{2} / 2\right)$ where $|z|^{2}=z_{1}^{2}+\cdots+z_{n-1}^{2}$;

(ii) Letting $v(z) d z_{1} \wedge \cdots \wedge d z_{n-1}$ be the expression for the volume form of $S^{n-1}$ with respect to these coordinates, then $v(0)=1$.

Lemma C.12 says that $z=\left(z_{1}, \ldots, z_{n-1}\right)$ are Morse coordinates for the functional $\omega \mapsto-\omega \cdot \frac{l}{|l|}$.

Proof of Lemma C.12. For $\omega=\left(y_{1}, \ldots, y_{n}\right) \in S_{+}^{n-1}(l)$, we have $\omega \cdot l=|l| y_{n}$. Since $y_{1}^{2}+\cdots+y_{n-1}^{2}=1-y_{n}^{2}$, we have $|z|^{2}=2\left(1-y_{n}\right)$ and thus $y_{n}=1-\frac{|z|^{2}}{2}$. Statement (i) follows.

With respect to the "standard" coordinates $\left(y_{1}, \ldots, y_{n-1}\right)$ on $S_{+}^{n-1}$, the volume form is given by $\frac{1}{y_{n}} d y_{1} \wedge \cdots \wedge$ $d y_{n-1}$. Since $z_{j}=\sqrt{\frac{2}{1+y_{n}}} y_{j}$, we have $d z_{j}=d y_{j}$ when $z=0$ (and $y_{n}=1$ ). Thus (ii) follows.

Define the functions $\widetilde{b}_{k, l}(x, z)$ by

$$
\widetilde{b}_{k, l}(x, z)=b_{k, l}(x, \omega(z)) v(z)
$$

where $v(z)$ is given as in Lemma C.12(ii). In view of Lemma C.12 (ii), $\widetilde{b}_{k, l}(x, 0)=b_{k, l}(x, l /|l|)$ for any $k, x$.

By the method of stationary phase (see, e.g., [Gr-S] pp. 19-24), one then gets for $l= \pm d$ and any given integer $M \geq 1$,

$$
\left.\int_{S_{+}^{n-1}(l)} d_{v o l} \omega e^{-i \eta \omega \cdot l} b_{k, l, \beta}(x, \omega) \stackrel{\eta \rightarrow+\infty}{=} \frac{e^{-i \eta|l|}}{\eta^{\frac{n-1}{2}}} \sum_{s=0}^{M-1} \frac{(2 \pi)^{\frac{n-1}{2}} e^{i \frac{\pi}{4}(n-1)}}{s !|l|^{s+\frac{n-1}{2}}(2 i)^{s}} \frac{1}{\eta^{s}} \Delta_{z}^{s}\right|_{z=0} \widetilde{b}_{k, l}(x, z)+O\left(\frac{1}{\eta^{M+\frac{n-1}{2}}}\right),
$$

where $\Delta_{z}:=\frac{\partial^{2}}{\partial z_{1}^{2}}+\cdots+\frac{\partial^{2}}{\partial z_{n-1}^{2}}$. (Here we are using the fact that $b_{k, l, \beta}=b_{k, l}$ on a neighborhood of $|l|$.) This leads to

$$
J_{l, R}(\eta) \stackrel{\eta \rightarrow+\infty}{=} \frac{e^{-i \eta|l|}}{(2 \pi)^{n-1}} \sum_{k+s=0}^{R-1+\left[\frac{n}{2}\right]} \frac{1}{\eta^{k+s-\frac{n-1}{2}}} \int_{\mathcal{F}} d x e^{-2 \pi i e_{l}(x)} \frac{(2 \pi)^{\frac{n-1}{2}} e^{i \frac{\pi}{4}(n-1)}}{s !|l|^{s+\frac{n-1}{2}}(2 i)^{s}} \Delta_{z \mid z=0}^{s} \widetilde{b}_{k, l}(x, z)+O\left(\frac{1}{\eta^{R}}\right)
$$

where $k$ and $s$ are understood to be nonnegative integers. For $m \geq 0$, define 


$$
C_{m}(x, l):=\left.\sum_{j=0}^{m} \frac{1}{j !}\left(\frac{1}{2 i|l|}\right)^{j} e^{-2 \pi i e_{l}(x)} \Delta_{z}^{j}\right|_{z=0} \widetilde{b}_{m-j, l}(x, z) .
$$

By setting $m=k+s$ in the asymptotic expansion above, we obtain:

C.15. Proposition. For $l= \pm d$,

$$
J_{l, R}(\eta) \stackrel{\eta \rightarrow+\infty}{=} \frac{(2 \pi)^{\frac{-n+1}{2}} e^{i \frac{\pi}{4}(n-1)}}{|l|^{\frac{n-1}{2}}} e^{-i \eta|l|} \sum_{m=0}^{R-1+\left[\frac{n}{2}\right]} \frac{1}{\eta^{m-\frac{n-1}{2}}} \int_{\mathcal{F}} C_{m}(x, l) d x+O\left(\frac{1}{\eta^{R}}\right) .
$$

C.16. THEOREM. Assume that $\mathcal{L}$ has nondegenerate length spectrum. Then for any $m \geq 0$ and any $l \in \mathcal{L} \backslash\{0\}$, the expression $C_{m, l}$ given by

$$
C_{m, l}:=\int_{\mathcal{F}} C_{m}(x, l) d x+\int_{\mathcal{F}} C_{m}(-x,-l) d x
$$

is a spectral invariant of $\Delta_{\varphi}+Q$.

The theorem follows from Propositions C.11 and C.15 and the fact that the fundamental domain $\mathcal{F}$ was chosen to be $\mathbb{Z}_{2}$-invariant.

C.18. Proposition. For $l \in \mathcal{L}$, we have in the notation of Theorem $C .16$ that

$$
C_{1}(x, l)=g(x, l)+\frac{i}{2} e^{-2 \pi i e_{l}(x)} a_{0}\left(|l|, x+l,-\frac{l}{|l|}\right) \int_{0}^{|l|} Q\left(x-\tau \frac{l}{|l|}\right) d \tau
$$

where $g(x, l)$ is a function independent of $Q$.

Proof. By Definition C.9 and Lemma A.18, $b_{0, l}$ is independent of $Q$. Thus, by (C.14), there exists a function $g_{1}(x, l)$ which does not depend on $Q$ such that

$$
C_{1}(x, l)=g_{1}(x, l)+e^{-2 \pi i e_{l}(x)} b_{1, l}\left(x, \frac{l}{|l|}\right) .
$$

Hence, by Definition C.6 and Equations (A.21), (A.24), (A.25), and (A.26), there exists a function $g(x, l)$ independent of $Q$ such that

$$
C_{1}(x, l)=g(x, l)+\frac{i}{2} e^{-2 \pi i e_{l}(x)} a_{0}\left(|l|, x+l,-\frac{l}{|l|}\right) \int_{0}^{|l|} Q\left(x+l-\tau \frac{l}{|l|}\right) d \tau
$$

The proposition follows since $Q$ is $\mathcal{L}$-periodic.

C.19. THEOREM. Assume that $\mathcal{L}$ has simple length spectrum and that the connection is $\mathbb{Z}_{2}$-invariant. Let $Q^{-}$ denote the odd part of $Q$; i.e., $Q^{-}=\frac{1}{2}(Q-\check{Q})$.Then in the notation of Theorem C.16

$$
C_{2, l}=-\frac{1}{4} \int_{\mathcal{F}} d x e^{-2 \pi i e_{l}(x)} a_{0}\left(|l|, x+l,-\frac{l}{|l|}\right)\left(\int_{0}^{|l|} d \tau Q^{-}\left(x-\tau \frac{l}{|l|}\right)\right)^{2}
$$

+ terms which do not involve $Q^{-}$.

Proof. In view of C.14, one has

$$
C_{2}(x, l)=\sum_{j=0}^{2} \frac{1}{j !}\left(\frac{1}{2 i|l|}\right)^{j} B_{j}(x, l)
$$

where

(C.21)

$$
B_{j}(x, l):=\left.e^{-2 \pi i e_{l}(x)} \Delta_{z}^{j}\right|_{z=0} \widetilde{b}_{2-j, l}(x, z) .
$$

Given any function $F(x, l)$, write $F^{+}(x, l)=\frac{1}{2}(F(x, l)+F(-x,-l))$. We have by Equation (C.17) that 
(C.22)

$$
C_{2, l}=2 \int_{\mathcal{F}} C_{2}^{+}(x, l) d x=\sum_{j=0}^{2} \frac{2}{j !}\left(\frac{1}{2 i|l|}\right)^{j} \int_{\mathcal{F}} B_{j}^{+}(x, l) d x .
$$

In the following, a function of any or all of the variables $x \in \mathbb{R}^{n}, l \in \mathcal{L}$ and $\omega \in S^{n-1}$ will be said to be even (respectively, odd) if it remains unchanged (respectively, is changed into its negative) when all the variables are replaced by their negatives. Note that in this sense, the function $(x, l) \mapsto e^{-2 \pi i e_{l}(x)}$ is even. By Proposition A.35, the function $(x, \omega) \mapsto a_{0}(t, x, \omega)$ is also even for any fixed $t \in \mathbb{R}$. Hence by Remark 2.32, the map $(x, \omega) \mapsto f_{1,0}(t, x, \omega)$ defined in (A.25) is even as well.

The functions $B_{j}$ in (C.21) are given as follows:

- $j=0$ : Using (C.8) with $k=2$ along with (A.27), (A.30), and the fact that $a_{1,0}$ and $a_{2,0}$ are independent of $Q$ (cf. (A.21), (A.25) and (A.31)), we have

$B_{0}(x, l)=e^{-2 \pi i e_{l}(x)}\left[a_{2,2}\left(|l|, x+l,-\frac{l}{|l|}\right)+a_{2,1}\left(|l|, x+l,-\frac{l}{|l|}\right)+\left.i(n-2) \frac{\partial}{\partial t}\right|_{t=|l|} a_{1,1}\left(t, x+l,-\frac{l}{|l|}\right)\right]$

+terms independent of $Q$.

- $j=1$ : By (C.8), (A.21), (A.24), (A.25), and (A.28),

$$
B_{1}(x, l)=\left.e^{-2 \pi i e_{l}(x)} \Delta_{z}\right|_{z=0} a_{1,1}(\omega \cdot l, x+l,-\omega(z)) v(z)+\text { terms independent of } Q
$$

with $v(z)$ as in Lemma C.12.

- $j=2$ : By (C.9), (A.19), and (C.21), $B_{2}(x, l)$ is independent of $Q$.

We will show below that $Q^{-}$contributes to $C_{2, l}$ only through the first term in $B_{0}^{+}(x, l)$ (the term involving $a_{2,2}$ ). Assuming this statement for now, we examine the dependence on $Q$ of the first term in the expression for $B_{0}(x, l)$,

Write $A_{2,2}(x, l)=a_{2,2}\left(|l|, x+l,-\frac{l}{|l|}\right)$ and $A_{0}(x, l)=a_{0}\left(|l|, x+l,-\frac{l}{|l|}\right)$. By (A.19), (A.21) and (A.34) we have

$$
A_{2,2}(x, l)=-\frac{1}{8} A_{0}(x, l)\left(\int_{0}^{|l|} Q\left(x-\tau \frac{l}{|l|}\right) d \tau\right)^{2} .
$$

Since $A_{0}(x, l)=A_{0}(-x,-l)$, we have

$$
A_{2,2}^{+}(x, l)=-\frac{1}{8} A_{0}(x, l)\left(\int_{0}^{|l|} Q^{-}\left(x-\tau \frac{l}{|l|}\right) d \tau\right)^{2}+\ldots
$$

where $\ldots$ is independent of $Q^{-}$. Since $e_{l}(x)=e_{-l}(-x)$, the theorem then follows from Equations (C.22) and (C.23).

It remains only to show that $Q^{-}$does not contribute to $C_{2, l}$ through $B_{1}^{+}(x, l)$ nor through the other terms in $B_{0}^{+}(x, l)$.

By (A.19), (A.21) and (A.32),

$$
a_{2,1}(t, x+l,-\omega)=\frac{i}{2} a_{0}(t, x+l,-\omega) \int_{0}^{t}\left(\frac{\square_{\varphi}\left(a_{0} f_{1,1}\right)}{a_{0}}+Q f_{1,0}\right)(\tau, x+l-(t-\tau) \omega,-\omega) d \tau .
$$

By (A.19) and (A.25), $a_{0}$ and $f_{1,0}$ are independent of $Q$, while by (A.26), $f_{1,1}$ depends linearly on $Q$. Moreover, for each fixed $t$, the functions $(x, \omega) \mapsto a_{0}(t, x, \omega)$ and $(x, \omega) \mapsto f_{1,0}(t, x, \omega)$ are both even. Thus $a_{2,1}(t, x+$ $l, \omega)+a_{2,1}(t,-x-l,-\omega)$ is independent of $Q^{-}$. Thus $Q^{-}$does not contribute to $B_{0}^{+}(x, l)$ through $a_{2,1}$. 
We next show that $Q^{-}$will not contribute to $C_{2, l}$ through $a_{1,1}$. At this stage, it is simpler to prove the stronger statement that $Q^{-}$does not contribute to the whole sum $J_{l, R}+J_{-l, R}$ through $a_{1,1}$. We come back to the expression for $J_{l, R}+J_{-l, R}$ obtained before using the method of stationnary phase. By (C.10), for any $l \neq 0$

$$
\begin{aligned}
J_{l, R}(\eta)+ & J_{-l, R}(\eta)=\frac{1}{(2 \pi)^{n-1}} \sum_{k=0}^{R+n-2} \eta^{n-1-k} \int_{\mathcal{F}} d x e^{-2 \pi i e_{l}(x)} \int_{S_{+}^{n-1}(l)} d_{v o l} \omega e^{-i \eta \omega \cdot l} b_{k, l, \beta}(x, \omega) \\
& +\frac{1}{(2 \pi)^{n-1}} \sum_{k=0}^{R+n-2} \eta^{n-1-k} \int_{\mathcal{F}} d x e^{2 \pi i e_{l}(x)} \int_{S_{+}^{n-1}(-l)} d_{v o l} \omega e^{i \eta \omega \cdot l} b_{k,-l, \beta}(x, \omega) .
\end{aligned}
$$

After changing $x$ into $-x$ and $\omega$ into $-\omega$ in the second sum and taking into account that $\mathcal{F}$ was chosen to be symmetric, one gets

$J_{l, R}(\eta)+J_{-l, R}(\eta)=\frac{1}{(2 \pi)^{n-1}} \sum_{k=0}^{R+n-2} \eta^{n-1-k} \int_{\mathcal{F}} d x e^{-2 \pi i e_{l}(x)} \int_{S_{+}^{n-1}(l)} d_{v o l} \omega e^{-i \eta \omega \cdot l}\left[b_{k, l, \beta}(x, \omega)+b_{k,-l, \beta}(-x,-\omega)\right]$.

By (A.26) and the fact that $Q$ is periodic,

$$
a_{1,1}(t, x+l,-\omega)=\frac{i}{2} a_{0}(t, x+l,-\omega) \int_{0}^{t} Q(x-\tau \omega) d \tau
$$

Thus

$$
a_{1,1}^{-}(t, x+l,-\omega)=\frac{i}{2} a_{0}(t, x+l,-\omega) \int_{0}^{t} Q^{-}(x-\tau \omega) d \tau
$$

where $a_{1,1}^{-}(t, x, \omega):=\frac{1}{2}\left(a_{1,1}(t, x, \omega)-a_{1,1}(t,-x,-\omega)\right)$ for all $t \in \mathbb{R}, x \in \mathbb{R}^{n}, \omega \in S^{n-1}$. In view of (C.7) and (C.26), we have to show that for any $s \geq 0$,

$$
\left.(x, l, \omega) \mapsto \frac{\partial^{s}}{\partial t^{s}}\right|_{t=\omega \cdot l} a_{1,1}^{-}(t, x+l,-\omega)
$$

is odd.

As the map $(x, l, \omega) \mapsto a_{0}(t, x+l,-\omega)$ is even for any $t \in \mathbb{R}$, it suffices to prove that for any $s \geq 0$, the function $g^{(s)}$ given by

is odd. Write

$$
g^{(s)}(x, l, \omega)=\left.\frac{\partial^{s}}{\partial t^{s}}\right|_{t=\omega \cdot l} \int_{0}^{t} Q^{-}(x-u \omega) d u
$$

$$
h_{(x, \omega)}(t)=\int_{0}^{t} Q^{-}(x-u \omega) d u .
$$

Then $h_{(-x,-\omega)}(t)=-h_{(x, \omega)}(t)$ for all $t$, so all the $t$-derivatives also satisfy $h_{(-x,-\omega)}^{(s)}(t)=-h_{(x, \omega)}^{(s)}(t)$ for all $t$. We thus have

$$
g^{(s)}(x, l, \omega)=h_{(x, \omega)}^{(s)}(\omega \cdot l)=-h_{(-x,-\omega)}^{(s)}(\omega \cdot l)=-g^{(s)}(-x,-l,-\omega) .
$$

I.e., $g^{(s)}$ is odd, thus completing the proof of Theorem C.19

We now assume that $n$ is even, say $n=2 m$, and that the Chern class of $L \rightarrow M$ is nondegenerate. By Remark 2.24 and Proposition 2.27, we may express the connection for the bundle $L \rightarrow M$ as a smooth real-valued 1-form $\varphi$ on $\mathbb{R}^{n}$ of the form

$$
\varphi=\varphi^{D}+\alpha+d^{\star} \psi
$$

where $\varphi^{D}$ is the distinguished connection, $\alpha$ is a harmonic 1-form, and $\psi$ is the pull-back to $\mathbb{R}^{n}$ of a smooth 2 -form on $M$. 
For any nonzero vector $l \in \mathcal{L}$, write

$$
\mathcal{G}_{l}: \mathbb{R}^{2 m} \rightarrow \mathbb{C}, x \mapsto \exp \left[-\left.\frac{2 \pi i}{|l|} \int_{0}^{|l|} d^{\star} \psi\right|_{\left(x-\tau \frac{l}{|l|}\right)}(l) d \tau\right] .
$$

(See Definition 2.17 for the meaning of $\left.d^{\star} \psi\right|_{\left(x-\tau \frac{l}{|l|}\right)}$ )

C.29. LemmA. Given $\varphi$ as in Equation (C.27), define $a_{0}$ as in Lemma A.18. For any $x \in \mathbb{R}^{2 m}$ and $l \in \mathcal{L}$, we have

$$
a_{0}(|l|, x+l,-l /|l|)= \pm e^{2 \pi i\left(e_{x}(l)-\alpha(l)\right)} \mathcal{G}_{l}(x)
$$

where the sign is given by $e^{i \pi e_{l}(l)}$.

Proof. By Lemma A.18, we have

$$
a_{0}\left(|l|, x+l,-\frac{l}{|l|}\right)=\exp \left[-\left.\frac{2 \pi i}{|l|} \int_{0}^{|l|} d \tau \varphi\right|_{\left(x+l-\tau \frac{l}{|l|}\right)}(l)\right] .
$$

Denote the right hand side of Equation (C.30) by $I_{\varphi}$. By Equation (C.27), we have

$$
a_{0}\left(|l|, x+l,-\frac{l}{|l|}\right)=I_{\varphi^{D}} I_{\alpha} I_{d^{\star} \psi} .
$$

Recall that for $w \in \mathbb{R}^{n}$, we have $\left.\varphi^{D}\right|_{w}(l)=-e_{w}(l)$; in particular, $\varphi^{D}$ depends linearly on $w$. Thus $\left.\varphi^{D}\right|_{\left(x+l-\tau, \frac{l}{|l|}\right)}(l)=-e_{x}(l)+\left(\frac{\tau-|l|}{|l|^{2}}\right) e_{l}(l)$ and

$$
I_{\phi^{D}}=e^{2 \pi i e_{x}(l)} \exp \left[\frac{-2 \pi i e_{l}(l)}{|l|^{2}} \int_{0}^{|l|}(\tau-|l|) d \tau\right]=e^{2 \pi i e_{x}(l)} e^{\pi i e_{l}(l)}= \pm e^{2 \pi i e_{x}(l)},
$$

where the last equality uses the fact that $e_{l}(l) \in \mathbb{Z}$ for all $l \in \mathcal{L}$.

Next, the harmonic 1-form $\alpha$ may be viewed as a linear functional on $\mathbb{R}^{n}$, and we have

$$
I_{\alpha}=e^{-2 \pi i \alpha(l)} \text {. }
$$

Finally, since $\psi$ is the pull-back of a 2 -form on $M$, it is $\mathcal{L}$-periodic. Hence

$$
I_{d^{*} \psi}=\mathcal{G}_{l}(x) .
$$

The lemma thus follows from Equation (C.31).

Proof of Proposition 4.1. The proposition follows from Theorem C.16, Propositions A.35 and C.18, and Lemma C.29.

\section{Appendix D. Proof of Proposition C.11.}

In this appendix we prove Proposition C.11 stated in Appendix C.

We fix $d \in \mathcal{L}$ and write $\beta$ for $\beta_{|d|}$.

Recall from Section A that $K=\frac{1}{2}\left(K_{+}+K_{-}\right)$. We first prove that the contribution of $K_{-}$to $\widehat{\beta W_{L}}(\eta)$ is $O\left(\eta^{-\infty}\right)$ as $\eta \rightarrow+\infty$. Indeed, as the symbol of $K_{-}(t, x+l, x)$ is given by $e^{i l \cdot \zeta-i t|\zeta|} a^{-}(t, x+l, \zeta)$ (see the beginning of Appendix $\mathrm{C}$ ), the sum

$$
\sum_{l= \pm d} \int_{\mathcal{F}} d x \int_{\mathbb{R}} d t e^{-i t \eta} \beta(t) K_{-}(t, x+l, x) e^{-2 \pi i e_{l}(x)}
$$

equals

$$
\frac{1}{(2 \pi)^{n}} \sum_{l= \pm d} \int_{\mathcal{F}} d x \int_{\mathbb{R}} d t e^{-i t \eta} \beta(t) e^{-2 \pi i e_{l}(x)} \int_{\mathbb{R}^{n}} d \zeta e^{i l \cdot \zeta-i t|\zeta|} a^{-}(t, x+l, \zeta)
$$




$$
=\frac{1}{(2 \pi)^{n}} \sum_{l= \pm d} \int_{\mathcal{F}} d x \int_{\mathbb{R}^{n}} d \zeta e^{i l \cdot \zeta} e^{-2 \pi i e_{l}(x)} \int_{\mathbb{R}} d t \beta(t) a^{-}(t, x+l, \zeta) e^{-i t(\eta+|\zeta|)},
$$

which, by integrations by parts with respect to $t$, is $O\left(\eta^{-\infty}\right)$ when $\eta \rightarrow+\infty$. Thus it suffices to consider $K_{+}$.

By the construction of $K_{+}^{N}$ (see Equation (A.4)) and regularity results of the Cauchy problem for the operator $\square_{\varphi}+Q$, we obtain for any $l \in \mathcal{L}$ the following property: for any $R \in \mathbb{N}$ there exists $N_{R} \in \mathbb{N}$ such that for any $N \geq N_{R}$ one can find $g_{R, N} \in C^{R}\left(\mathbb{R} \times \mathbb{R}^{n} \times \mathbb{R}^{n}\right)$ with the property that

$$
\begin{gathered}
\int_{\mathcal{F}} d x \int_{\mathbb{R}} d t e^{-i t \eta} \beta(t) K_{+}(t, x+l, x) e^{-2 \pi i e_{l}(x)}=\int_{\mathcal{F}} d x \int_{\mathbb{R}} d t e^{-i t \eta} \beta(t) K_{+}^{N}(t, x+l, x) e^{-2 \pi i e_{l}(x)} \\
+\int_{\mathcal{F}} d x \int_{\mathbb{R}} d t e^{-i t \eta} \beta(t) g_{R, N}(t, x+l, x) e^{-2 \pi i e_{l}(x)} .
\end{gathered}
$$

It follows that for any $R \in \mathbb{N}$ there exists $N_{R} \in \mathbb{N}$ such that for any $N \geq N_{R}$

$$
\begin{gathered}
\widehat{\beta W_{L}}(\eta) \stackrel{\eta \rightarrow+\infty}{=} \sum_{l= \pm d} \frac{1}{2} \int_{\mathcal{F}} d x \int_{\mathbb{R}} d t e^{-i t \eta} \beta(t) K_{+}^{N}(t, x+l, x) e^{-2 \pi i e_{l}(x)}+O\left(\eta^{-R}\right) \\
\stackrel{\eta \rightarrow+\infty}{=} \sum_{l= \pm d} \frac{1}{2} \frac{1}{(2 \pi)^{n}} \int_{\mathcal{F}} d x \int_{\mathbb{R}^{n}} d \zeta \int_{\mathbb{R}} d t e^{-i t \eta} e^{-2 \pi i e_{l}(x)} e^{i l \cdot \zeta+i t|\zeta|} \sum_{j=0}^{N} \beta(t) a_{j}^{+}(t, x+l, \zeta) \chi(|\zeta|)+O\left(\eta^{-R}\right)
\end{gathered}
$$

Hence

$$
\widehat{\beta W_{L}}(\eta) \stackrel{\eta \rightarrow+\infty}{=} \frac{1}{2}\left(I_{d}(\eta)+I_{-d}(\eta)\right)+O\left(\eta^{-R}\right)
$$

where, using notation (A.16) and the fact that $a_{j}^{+}$is postiive-homogeneous of degree $-j$ in the third variable, $I_{l}(\eta)$ is given by

$$
\frac{1}{(2 \pi)^{n}} \int_{\mathcal{F}} d x e^{-2 \pi i e_{l}(x)} \int_{S^{n-1}} d_{\text {vol }} \omega \int_{\mathbb{R}_{+}} d \rho \rho^{n-1} \chi(\rho) e^{i \rho \omega \cdot l} \int_{\mathbb{R}} d t e^{-i t(\eta-\rho)} \beta(t) \sum_{j=0}^{N} \frac{a_{j}(t, x+l, \omega)}{\rho^{j}} .
$$

with $N \geq N_{R}$. Denoting by $\widehat{\beta a_{j}}$ the Fourier transform of $\beta a_{j}$ with respect to the variable $t$, we then obtain for $l= \pm d$

$$
I_{l}(\eta)=\frac{1}{(2 \pi)^{n}} \int_{\mathcal{F}} d x e^{-2 \pi i e_{l}(x)} \int_{S^{n-1}} d_{v o l} \omega \sum_{j=0}^{N} \int_{\mathbb{R}} d \rho \rho^{n-1-j} \chi(\rho) e^{i \rho \omega \cdot l} \widehat{\beta a_{j}}(\eta-\rho, x+l, \omega)
$$

where we used the fact that $\chi(\rho)=0$ for $\rho \leq \frac{1}{2}$ (see (A.6).) Setting $\tau:=\eta-\rho$, we have

$$
I_{l}(\eta)=\frac{1}{(2 \pi)^{n}} \int_{\mathcal{F}} d x e^{-2 \pi i e_{l}(x)} \int_{S^{n-1}} d_{v o l} \omega e^{i \eta \omega \cdot l} \sum_{j=0}^{N} \int_{\mathbb{R}} d \tau e^{-i \tau \omega \cdot l}(\eta-\tau)^{n-1-j} \chi(\eta-\tau) \widehat{\beta a_{j}}(\tau, x+l, \omega) .
$$

Note that the integral with respect to $\tau$ in the latter expression is actually an integral over the interval $]-\infty, \eta-1 / 2]$, as $\operatorname{supp}(\chi) \subset[1 / 2,+\infty[$.

In order to expand the expression $(\eta-\tau)^{n-1-j}$, recall that the binomial coefficients $\left(\begin{array}{c}-m \\ k\end{array}\right)$ are defined when $m$ and $k$ are positive by

$$
\left(\begin{array}{c}
-m \\
k
\end{array}\right)=\frac{-m(-m-1) \ldots(-m-k+1)}{k !}=(-1)^{k}\left(\begin{array}{c}
m+k-1 \\
k
\end{array}\right)
$$

and that $\left(\begin{array}{c}-m \\ 0\end{array}\right)=1$. For $p$ a negative integer, the power series expansion for $(a+b)^{p}=a^{p}\left(1+\frac{b}{a}\right)^{p}$ is then given by the binomial expansion

$$
(a+b)^{p}=\sum_{k=0}^{\infty}\left(\begin{array}{l}
p \\
k
\end{array}\right) a^{p-k} b^{k}
$$


Interpreting $\left(\begin{array}{l}p \\ k\end{array}\right)$ to be zero when $p$ is a nonnegative integer and $k>p$, then the expansion (D.3) is valid for all integers $p$.

Since $\eta \pm \tau$ is the symbol of the differential operator $\eta \pm i \frac{d}{d t}$, the operator with symbol $(\eta \pm \tau)^{p}$, denoted formally by $\left(\eta \pm i \frac{d}{d t}\right)^{p}$, is given by

$$
\left(\eta \pm i \frac{d}{d t}\right)^{p}=\sum_{k=0}^{\infty}\left(\begin{array}{l}
p \\
k
\end{array}\right) \eta^{p-k}\left( \pm i \frac{d}{d t}\right)^{k}
$$

We then have the following expression for $I_{l}(\eta)$ :

(D.5)

$$
\left.\frac{1}{(2 \pi)^{n}} \int_{\mathcal{F}} d x e^{-2 \pi i e_{l}(x)} \int_{S^{n-1}} d_{v o l} \omega e^{i \eta \omega \cdot l} \sum_{j=0}^{N}\left(\eta-i \frac{d}{d t}\right)^{n-1-j}\right|_{t=\omega \cdot l} \int_{\mathbb{R}} d \tau e^{-i \tau t} \chi(\eta-\tau) \widehat{\beta a_{j}}(\tau, x+l, \omega) .
$$

For $f \in C_{0}^{\infty}(\mathbb{R})$, Equation (D.4) implies that

$$
\left(\eta \pm i \frac{d}{d t}\right)^{n-1-j}(f)=\sum_{r=0}^{R+n-2-j}\left(\begin{array}{c}
n-1-j \\
r
\end{array}\right) \eta^{n-1-j-r}\left( \pm i \frac{d}{d t}\right)^{r}(f)+O\left(\eta^{-R}\right)
$$

when $\eta \rightarrow+\infty$.

As $\beta \in C_{0}^{\infty}(\mathbb{R})$, the Fourier transform of $\beta(\cdot) a_{j}(\cdot, x+l, \omega)$ is in $\mathcal{S}(\mathbb{R})$ for any $x \in \mathbb{R}^{n}, l \in \mathcal{L}$ and $\omega \in S^{n-1}$. It follows that for any integer $k \geq 1$ there exists a constant $C=C(k, l)>0$ such that for any $\tau \in \mathbb{R}, x \in \mathcal{F}$, and $\omega \in S^{n-1}$,

$$
|\tau|^{k-1}\left|\widehat{\beta a_{j}}(\tau, x+l, \omega)\right| \leq C .
$$

Thus for any $r \in \mathbb{N}$ and $l= \pm d$, it follows from the fact that $\chi(s)=1$ for $s \geq 1$ (see A.6) that

$$
\begin{gathered}
\left.\frac{d^{r}}{d t^{r}}\right|_{t=\omega \cdot l} \int_{\mathbb{R}} d \tau e^{-i \tau t}(1-\chi(\eta-\tau)) \widehat{\beta a_{j}}(\tau, x+l, \omega) \\
\left.\stackrel{\text { definition of } \chi}{=} \frac{d^{r}}{d t^{r}}\right|_{t=\omega \cdot l} \int_{\eta-1}^{+\infty} d \tau e^{-i \tau t}(1-\chi(\eta-\tau)) \widehat{\beta a_{j}}(\tau, x+l, \omega) \stackrel{\eta \rightarrow+\infty}{=} O\left(\eta^{-\infty}\right)
\end{gathered}
$$

uniformly in $x \in \mathcal{F}$ and $\omega \in S^{n-1}$. Hence, in view of (D.5), $I_{l}(\eta)$ admits for $\eta \rightarrow+\infty$ the asymptotic expansion $\left.\frac{1}{(2 \pi)^{n}} \int_{\mathcal{F}} d x e^{-2 \pi i e_{l}(x)} \int_{S^{n-1}} d_{v o l} \omega e^{i \eta \omega \cdot l} \sum_{j=0}^{N}\left(\eta-i \frac{d}{d t}\right)^{n-1-j}\right|_{t=\omega \cdot l} \int_{\mathbb{R}} d \tau e^{-i \tau t} \widehat{\beta a_{j}}(\tau, x+l, \omega)+O\left(\eta^{-\infty}\right)$.

The integral $\frac{1}{2 \pi} \int_{\mathbb{R}} d \tau e^{i \tau t} \widehat{\beta a_{j}}(\tau, x+l, \omega)$ is the inverse Fourier transform of $\widehat{\beta a_{j}}$. Hence for $\eta \rightarrow+\infty, I_{l}(\eta)$ equals

$$
\left.\frac{1}{(2 \pi)^{n-1}} \int_{\mathcal{F}} d x e^{-2 \pi i e_{l}(x)} \int_{S^{n-1}} d_{v o l} \omega e^{i \eta \omega \cdot l} \sum_{j=0}^{N}\left(\eta-i \frac{d}{d t}\right)^{n-1-j}\right|_{t=\omega \cdot l} \beta(-t) a_{j}(-t, x+l, \omega)+O\left(\eta^{-\infty}\right)
$$

or, changing $t$ into $-t$ and $\omega$ into $-\omega$, this equals

$$
\left.\frac{1}{(2 \pi)^{n-1}} \int_{\mathcal{F}} d x e^{-2 \pi i e_{l}(x)} \int_{S_{+}^{n-1}(l)} d_{v o l} \omega e^{-i \eta \omega \cdot l} \sum_{j=0}^{N}\left(\eta+i \frac{d}{d t}\right)^{n-1-j}\right|_{t=\omega \cdot l} \beta(t) a_{j}(t, x+l,-\omega)+O\left(\eta^{-\infty}\right)
$$

(we integrate on $S_{+}^{n-1}(l)$ instead of $S^{n-1}$ because $\operatorname{supp}(\beta) \subset \mathbb{R}_{+}$).

In Equation (D.6), make the change of index $k=j+r$ to obtain

$$
\left(\eta \pm i \frac{d}{d t}\right)^{n-1-j}(f)=\sum_{k=j}^{R+n-2}\left(\begin{array}{c}
n-1-j \\
k-j
\end{array}\right) \eta^{n-1-k}\left( \pm i \frac{d}{d t}\right)^{k-j}(f)+O\left(\eta^{-R}\right)
$$


Denote by $g_{j}$ the function $t \mapsto \beta(t) a_{j}(t, x+l,-\omega)$ (with $x, l$ and $\omega$ being fixed). By interchanging the order of summation, we have for any integer $R$ such that $N \geq R+n-2$,

$$
\left.\left.\sum_{j=0}^{N}\left(\eta+i \frac{d}{d t}\right)^{n-1-j}\right|_{t=\omega \cdot l} g_{j}(t) \stackrel{\eta \rightarrow+\infty}{=} \sum_{k=0}^{R+n-2} \sum_{j=0}^{k}\left(\begin{array}{c}
n-1-j \\
k-j
\end{array}\right) \eta^{n-1-k}\left(i \frac{d}{d t}\right)^{k-j}\right|_{t=\omega \cdot l} g_{j}(t)+O\left(\eta^{-R}\right)
$$

where the error term is uniform in $x \in \mathcal{F}$ and $\omega \in S^{n-1}$. Thus

$$
I_{l}(\eta) \stackrel{\eta \rightarrow+\infty}{=} \frac{1}{(2 \pi)^{n-1}} \int_{\mathcal{F}} d x e^{-2 \pi i e_{l}(x)} \int_{S_{+}^{n-1}(l)} d_{v o l} \omega e^{-i \eta \omega \cdot l} \sum_{k=0}^{R+n-2} b_{k, l, \beta}(x, \omega) \eta^{n-1-k}+O\left(\eta^{-R}\right),
$$

where $b_{k, l, \beta}(x, \omega)$ is given in Definition C.6. Proposition C.11 now follows from Equations (D.1) and (D.7) .

\section{REFERENCES}

[D-G] J. Duistermaat, V. Guillemin. The spectrum of positive elliptic operators and periodic bicharacteristics. Invent. Math. 29 (1975) $39-79$.

[D] J. L. Dupont. Curvature and Characteristic Classes. Lecture Notes in Mathematics, 640, Springer-Verlag.

[E] G. Eskin. Inverse spectral problem for the Schrödinger equation with periodic vector potential. Commun. Math. Phys. 125, No. 2 (1989) 263-300.

[E-R-T] G. Eskin, J. Ralston and E. Trubowitz. On Isospectral Periodic Potentials in $\mathbb{R}^{n}$, I, II. Comm. in Pure and Appl. Math. 37 (1984) 647-676, 715-753.

[Gi] P. B. Gilkey. Recursion relations and the asymptotic behavior of the eigenvalues of the Laplacian. Comp. Math., 38, No. 2 (1978) $201-240$.

[Go-K1] C. Gordon and T. Kappeler. On isospectral potentials on tori. Duke Math. J. 63, No. 1 (1991) 217-233.

[Go-K2] C. Gordon and T. Kappeler. On isospectral potentials on flat tori II. Comm. Partial Diff. Eqs. 20 (1995) 709-728.

[GW] C. Gordon and E. N. Wilson, The spectrum of the Laplacian on Riemannian Heisenberg manifolds. Mich. Math. J. 33 (1986) $253-271$.

[GH] P. Griffiths and J. Harris. Principles of Algebraic Geometry. John Wiley \& Sons, 1978.

[Gr-S] A. Grigis and J. Sjöstrand. Microlocal Analysis for Differential Operators. London Mathematical Society, Lecture Note Series 196 , Cambridge University Press.

[G] V. Guillemin. Inverse spectral results on two-dimensional tori. J. Am. Math. Soc. 3, No. 2, (1990) 375-387.

[K] T. Kappeler. On isospectral periodic potentials on a discrete lattice II. Adv. in Appl. Math. 9 (1988) 428-438.

[Ku] P. Kuchment. Floquet theory for partial differential equations. Birkhäuser, 1993.

[La] P. Lax. Asymptotic solutions of oscillatory initial value problems. Duke Math. J. 24 (1957) 624-646.

[R] J. Roe. Elliptic operators, topology, and asymptotic methods. Pitman Research Notes in Mathematics, 395, Addison Wesley Longman, 1998.

Carolyn S. Gordon \& David L. Webb

Department of Mathematics, Dartmouth College

6188 Bradley Hall, Hanover, NH 03755-3551

e-mail: carolyn.s.gordon@dartmouth.edu,david.1.webb@dartmouth.edu

Pierre Guerini \& Thomas Kappeler

Institut für Mathematik, Universität Zürich-Irchel

Winterthurerstrasse 190, CH-8057 Zürich

e-mail: pguerini@math.unizh.ch,tk@math.unizh.ch 